\title{
On Magnitude Determination by Using Macroseismic Data
}

\author{
A. G. Galanopotlos (*)
}

Ricevuto il 16 Giugno 1961

\section{INTRODUCTION.}

The time interval between two seismic periods in a given region may fluctuate within very wide limits. Quite often this interval takes over 50 years and not rarely over 1 and 2 centuries or more. This implies that the measure which determines the degree of the earthquake activity in a given region can not be reliable, if it is not based on data rovering a period of at least 100 years.

It was already proxed that the most reliable data for measuring the seismicity of a given region are the magnitudes. Since the magnitude determination is connected with the existence of seismic records, the magnitude of shocks having occurred before the operation of modern seismographs, i. e. before 1900 , cannot be determined in a regular way. The need for magnitude data covering very large periods led several seismologists to relate the macroseismic data with the magnitude. However, the relations established between the magnitude and the macrosəismic data all have the general form:

$$
M=b I o+k \log h+c
$$

In the formulae proposed the coefficients $b, k$, and $c$ have values 0.5 to $0.8,1.0$ to 2.35 and -1.48 to 0.4 , respectively (Karnik, 1960). A

(*) Acknowledgments - The author is indebted to Mr. J. Abdelides, assistant of the Seismological Laboratory, Athens University, for his help in checking the statistical processes. The author is also grateful to Dr. M. Batth and Dr. V. Karnik for valuable suggestions and eriticism and much helpful discussion by correspondence. 
simple analysis of the formulae proposed shows that a slight change of the depth, $h$, results in a significant change in the magnitude, M. Further, the depth is usually unknown and the depth determinations are not very accurate, especially when macroseismic data are used. In shallow shocks the error may be quite large. Even with instrumental data, for shocks at a depth of $16 \mathrm{~km}$ the error may well be $\pm 6 \mathrm{~km}$, in favorable cases. At a depth of $50 \mathrm{~km}$ it may be as high as $+20 \mathrm{~km}$ (BenioffGutenberg, 1955).

The relation of the intensity, $I$, to the acceleration of the shock, $a$, is given by different empirical formulae having the general form:

$$
I=p \log a+q
$$

where $p$ and $q$ have values 2 to 3 and 1.5 to 2.5 , respectively (Karnik, 1956). These differences in the values of the coefficients $p$ and $q$ has an unfavorable influence on the macroseismic determination of the $\mathbf{M}$. Moreover, most of the shocks have their epicentral tract in unpopulated areas or offshore. In these cases an error of 2 degrees in the estimation of the epicentral intensity is not rare. The corresponding error in the determination of the magnitude is therefore at least 1 magnitude unit.

\section{EQUATION USED.}

The present investigation is based on a simple empirical equation. According to B. Gutenberg and C. F. Richter, (1942):

$$
\frac{a D^{2}}{i t}=\text { const. } V \bar{E} \text {. }
$$

Since

$$
a_{0} h^{2}=a D^{2}=a_{r} R^{2}
$$

it can be written:

$$
\begin{gathered}
a_{0} h=\text { const. } / \bar{E} \\
\frac{a_{r} R^{2}}{h}=\text { const. } \sqrt{E} .
\end{gathered}
$$

Combining [5] and [6], there results:

$$
a_{r} a_{0} R^{2}=C E
$$


At the limit of perceptibility we may assume that the hypocentral distance $R$ is approximately equal to the epicentral distance $r$. Thus we may put $\pi R^{2} \cong \pi r^{2}=A$ (= Area over which the shock was felt). From field observations we are convinced that the macroseismic effects " must correspond only to that level of acceleration which persisted long enough to produce permanent effects, which must be less than the maximum acceleration recorded on a complete seismogram" (Gutenberg-Richter, 1942; Byerley, 1942). Taking this into consideration and that the empirical relation

$$
\log a=\mathrm{I} / 3-1 / 2
$$

"fails for higher intensities" (Gutenberg-Richter, 1956a), it may be assumed that the acceleration at the epicenter $a_{0} \cong c I o$. This assumption( $\left.{ }^{*}\right)$ is furthermore justified in view of other much larger uncertainties involved in the estimation of the macroseismic elements of the shocks, especially those of the past centuries, and more over it is well-substantiated by the results obtained. Assuming now that the acceleration at the limit of perceptibility $a_{r} \cong$ constant, and that $E-10^{\mathrm{k} 1}+\mathrm{k} 2 \mathrm{M}$, we arrive at

$$
\mathrm{M}=C_{1} \log A I_{0}+C_{2} \text {. }
$$

It was found by B. Gutenberg (1945), that the energies released in the longitudinal and transverse waves of an earthquake are about equal, regardless of focal depth. More recently, however, Gutenberg and Richter (1956b) assume the energy in $P$ waves to be only half that in $S$ waves. Later it was found by M. Băth (1958), that the energy ratio $E_{S} / E_{P}=1.5 \pm 0.4$ is independent of magnitude and of epicentral intensity. Outside the meizoseismal area the macroseismic effects are not due to surface waves, but to body waves, and mostly to shear waves (Sieberg, 1932; Benioff-Gutenberg, 1955). Curves giving equal values of $Q$ for $S$ as a function of epicentral distance $\Delta$ and focal depth $h$ show very little change with depth in the range of felt shaking (GutenbergRichter, 1956b). "With increasing focal depth, less energy arrives

(*) The assumption $a \cong c I o$ differs from the basic relation $\log a \cong$ cIo used in all macroseismic computations. However, this assumption is widely used in the standards for reducing the numerical values of the horizontal coefficient of the seismic foree in the Building Codes in earthquakeprone countries, with reference to the class of the area or the kind of ground. (" Earthquake-Resistant Regulations of the World ", compiled by Organizing Committee, SWCEE, 1960). 
near the epicenter more at greater distance" (Gutenberg, 1945). It is well known that "A shallow shock may be heavily felt over a small area, but the effects do not extend very far. A deep shock gives a moderate shaking to a much greater area" (Eiby, 1957). From equations [5] and [6] it is obvious that the epicentral intensity, $I_{0}$, is almost
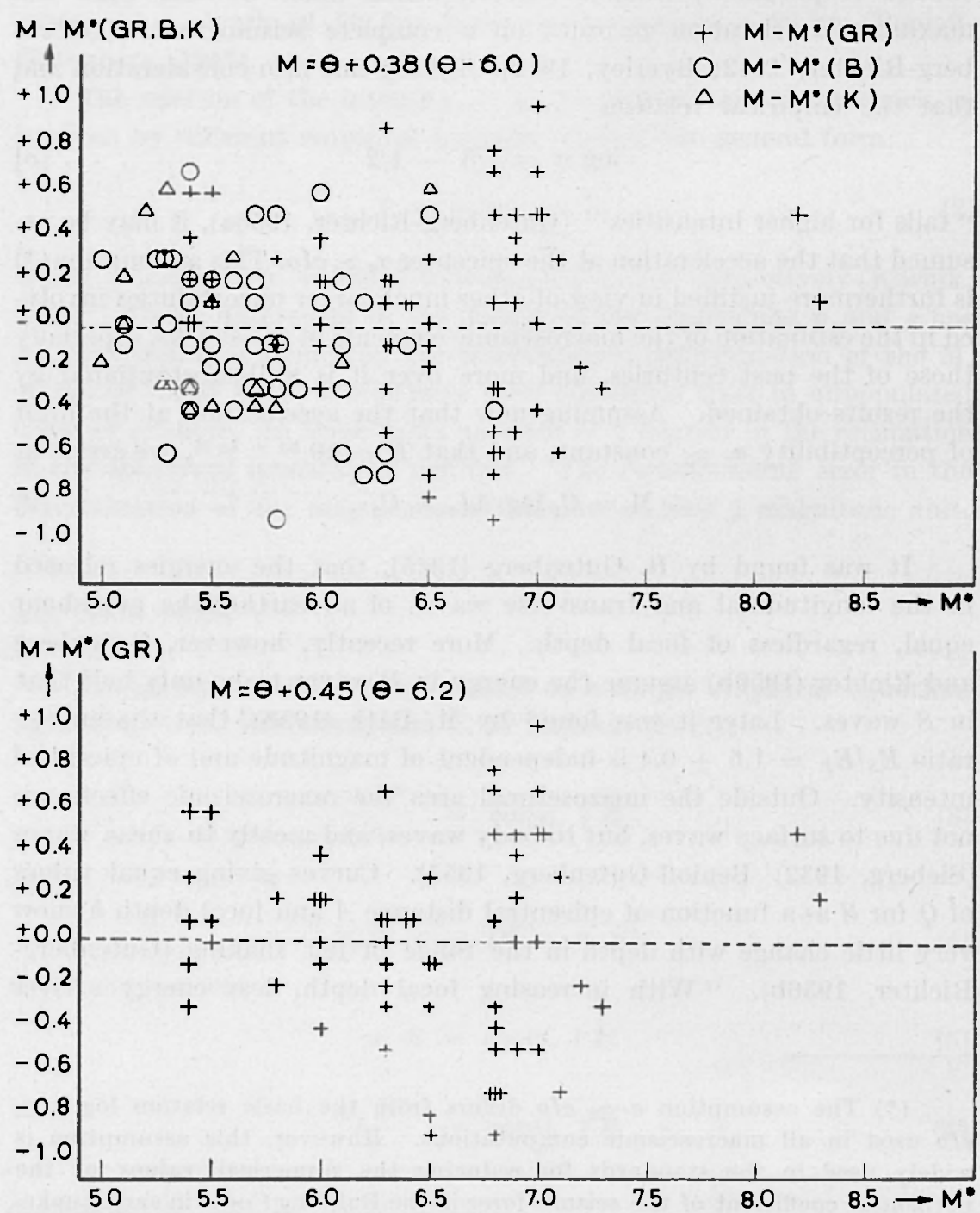

Fig. 1. - Variations with $M^{*}$ of differences between macroseismic magnitudes computed from Eqs. [10] and [12] and instrumental magnitudes $M^{*}(\mathrm{GR}, \mathrm{B}, \mathrm{K})$ and $M^{*}(\mathrm{GR})$, respectively. 
inversely proportional to $h$, but the area of perceptibility, $A$, is approximately proportional to $h$. As the wave velocity increases and the coefficient of absorption decreases with increasing depth, the distances up to which the earthquake energy is felt increase with the focal depth (Byerly-Denoyer, 1958; Sponheuer, 1960). Thus the product $A I_{0}$ of

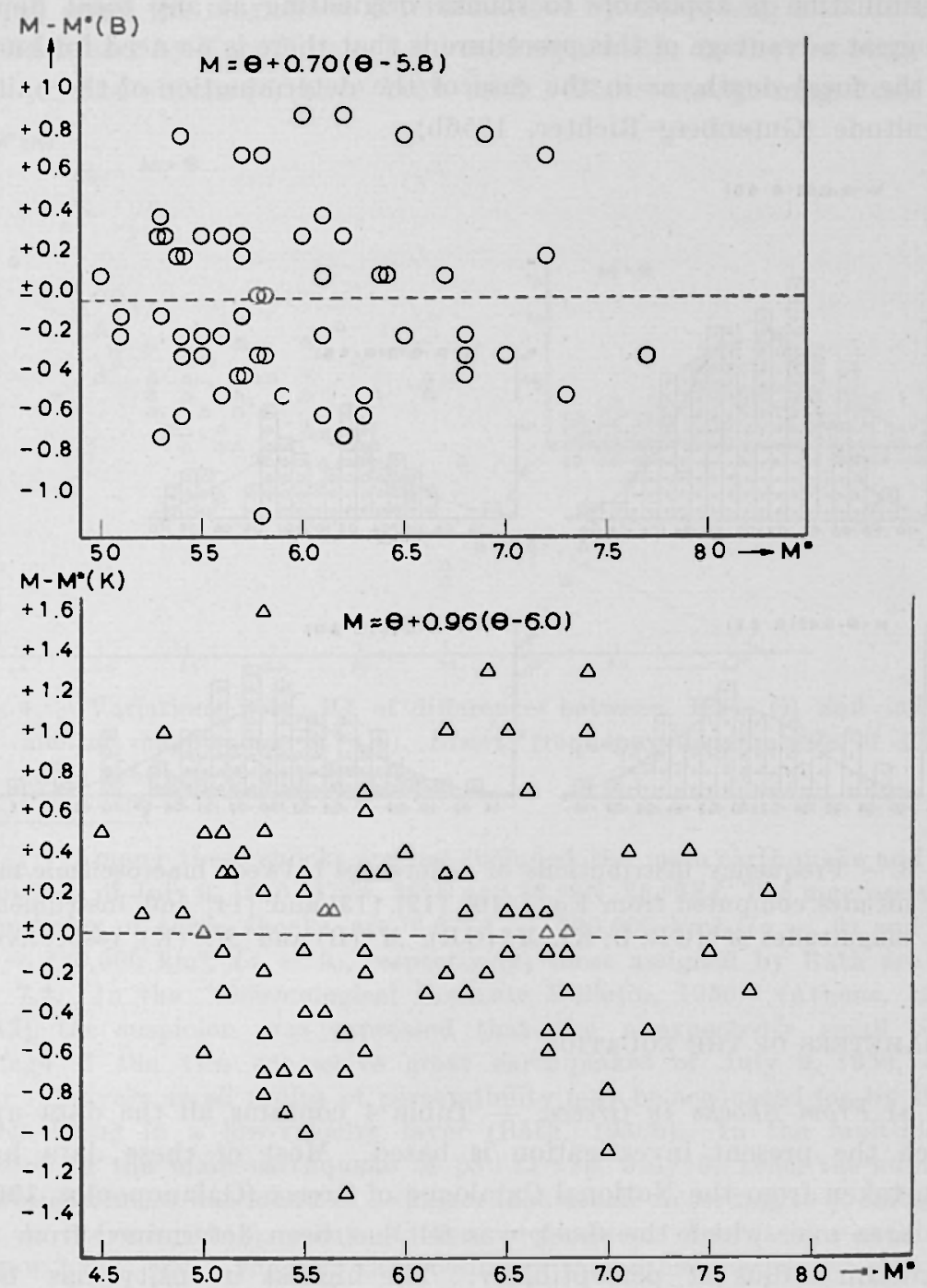

Fig. 2. - Variations with $M^{*}$ of differences between macroseismic magnitudes computed from Eqs. [13] and [14] and instrumental magnitudes $M^{*}(\mathrm{~B})$ and $M^{*}(\mathrm{~K})$, respectively. 
earthquakes with the same $E$ remains practically constant for any focal depth (Toperczer, 1953, 1960). This means that the quantity $\log A I_{0}$, used for the determination of earthquake magnitudes, has the advantage of being the same for two shocks of the same magnitude originating at different depths. This explains why the proposed procedure for $\boldsymbol{M}$ determination is applicable to shocks originating at any focal depth. The great advantage of this procedure is that there is no need for knowing the focal depth, as in the case of the determination of the unified magnitude (Gutenberg-Richter, 1956b).
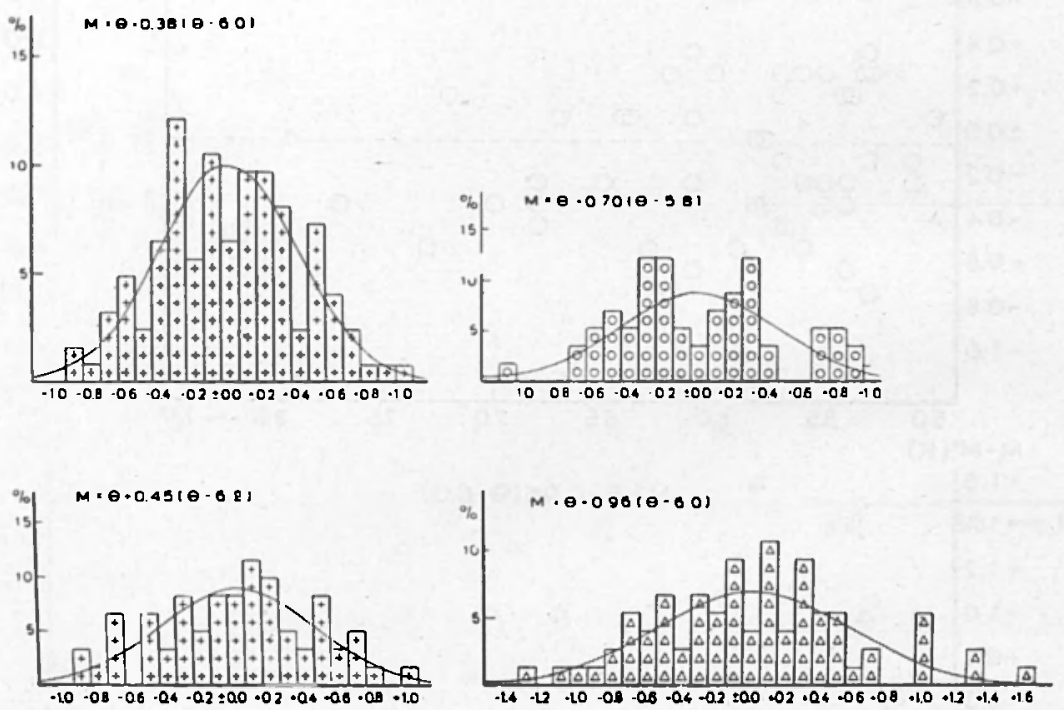

Fig. 3. - Frequency distributions of differences between macroseismic magnitudes computed from Eqs. [10], [12], [13] and [14] and instrumental magnitudes $M^{*}(\mathrm{GR}, \mathrm{B}, \mathrm{K}), I^{*}(\mathrm{GR}), M^{*}(\mathrm{~B})$ and $M^{*}(\mathrm{~K})$, respectively.

\section{Parameters of the equation.}

a) From Shocks in Greece. - Table 4 contains all the data upon which the present investigation is based. Most of these data have been taken from the National Catalogue of Greece (Galanopoulos, 1960). The area over which the shock was felt has been determined from the maximum radius of perceptibility. The highest intensity has been adopted as epicentral intensity The magnitudes and the depths of the shocks have been taken from Gutenberg and Richter (1954), Gutenberg (1956, 1959), Richter (1958), Båth (1956a), Karnik (1956) and station bul- 
letins. All the magnitudes assigned by Gutenberg and Richter have been used. The magnitudes computed by Karnik have been used for lack of magnitude determinations by Båth. Where no magnitude was available, the average of the magnitudes assigned by Båth (1956a) from both body waves and surface waves has been adopted as the value of $M$. In the second stage the magnitude determinations by Gutenberg and Richter, by Båth and by Karnik have been used separately. In the first stage the data of 124 shocks $\left(^{*}\right)$ have been used. There are 61 magnitude de-

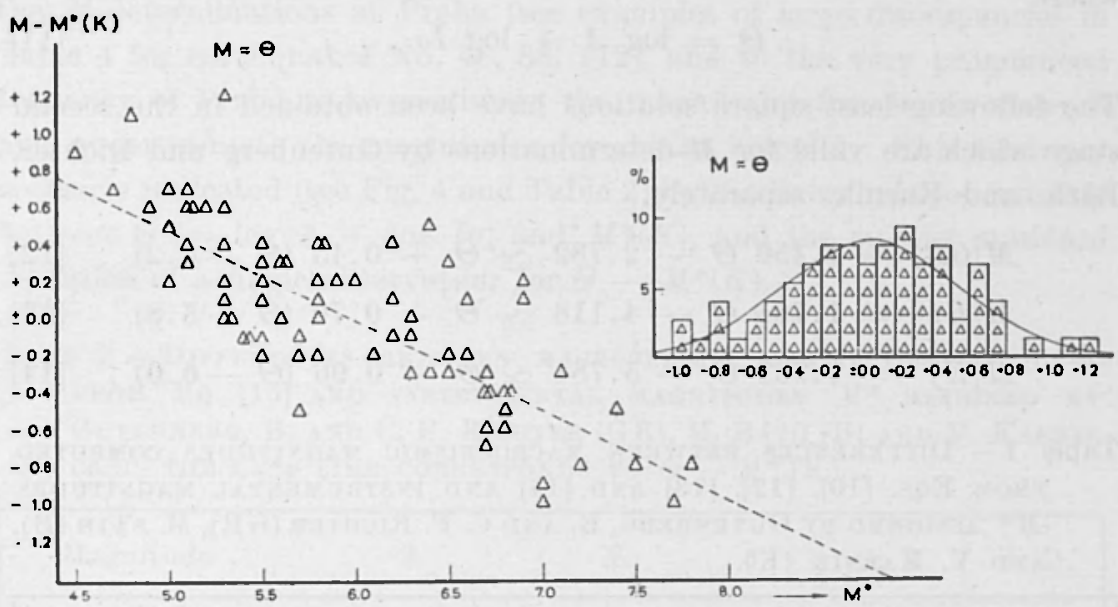

Fig. 4. - Variations with $M^{*}$ of differences between $M(=\Theta)$ and instrumental magnitudes $M^{*}(\mathrm{~K})$. Insert, frequency distributions of differences between $\mathrm{M}$ and $M^{*}(\mathrm{~K})$.

$(*)$ Among these shocks are not included the main earthquake and the aftershock of July $9,1956\left(37^{\circ} \mathrm{N}, 26^{\circ} \mathrm{E}\right.$ and $\left.36.08 \mathrm{~N}, 25 .^{\circ} 2 \mathrm{E}\right)$. The macroseismic magnitudes of these shocks are $6.6\left(A=300,000 \mathrm{~km}^{2}\right.$, Io $\left.=9\right)$ and 6.3 $\left(A=180,000 \mathrm{~km}^{2}\right.$, Io $=9$ ), respectively; those assigned by Båth are 7.7 and 7.2. In the "Seismological Institute Bulletin, 1956" (Athens, 1957, p. 43), the suspicion was expressed that the unexpectedly small total damage of the two successive great earthquakes of July 9, 1956, and their relatively small radius of perceptibility may be accounted for by their source being in a low-velocity laver (Batth, 1956b). In the fault-plane solution of the main earthquake of $03: 11: 39$, July 9, 1956, the number of inconsistencies was found to be higher than usual. According to J. Hodgson and A. Stevens (1958), many of these inconsistent observations are in a narrow band, which suggests the possibility that other mechanism may be operating. Such being the case, we have decided to ecxlude these shocks from the present investigation, although the discrepancies of their magnitudes ( -1.1 and -0.9 , respectively) are within the limits of error of $\boldsymbol{M}$. determinations from instrumental data (Båth, 1956a). 
terminations by Gutenberg and Richter, 58 by Båth and 75 by Karnik.

Plotting $\Theta-M^{*}$ as a function of $M^{*}$ (= magnitude computed from instrumental data) gives a straight line passing through the axis of zero residuals around $M^{*}=6.0$. The data are very well represented by the resulting, purely empirical equation:

$$
M(G R, B, K)=1.385 \Theta-2.315 \cong \Theta+0.38(\Theta-6.0),
$$

where

$$
\Theta=\log A+\log I o \text {. }
$$

The following least-square solutions have been obtained in the second stage which are valid for $M$-determinations by Gutenberg and Richter, Båth, and Karnik, separately,

$$
\begin{aligned}
& M(G R)=1.450 \Theta-2.782 \cong \Theta+0.45(\Theta-6.2) \\
& M(B)=1.704 \Theta-4.118 \cong \Theta+0.70(\Theta-5.8) \\
& M(K)=1.961 \Theta-5.784 \cong \Theta+0.96(\Theta-6.0)
\end{aligned}
$$

\begin{tabular}{|c|c|c|c|c|c|}
\hline Magnitudes & $h$ & $N$ & $\bar{\delta}$ & S. E. & S. D. \\
\hline$M^{*}(\mathrm{GR}, \mathrm{B}, \mathrm{K})$ & $n,(n),>n$ & 124 & -0.01 & \pm 0.04 & \pm 0.40 \\
\hline$M^{*}(\mathrm{GR})$ & $n,(n),>n$ & 61 & +0.01 & \pm 0.06 & \pm 0.45 \\
\hline$M *(\mathrm{~B})$ & $n,>n$ & 58 & -0.01 & \pm 0.06 & \pm 0.46 \\
\hline$M *(\mathrm{~K})$ & $n,(n)>, n$ & 75 & +0.01 & \pm 0.07 & \pm 0.58 \\
\hline \multicolumn{6}{|c|}{$\begin{array}{l}h=\text { depth of foci of earthquakes used }(n=\text { normal, }(n)=\text { slightly } \\
\text { below normal, }>n=\text { deep); }\end{array}$} \\
\hline \multicolumn{6}{|c|}{$N=$ number of earthquakes used; } \\
\hline \multicolumn{6}{|c|}{$\bar{\delta}=$ mean difference; } \\
\hline \multicolumn{6}{|c|}{ S. E. = standard error of the mean; } \\
\hline \multicolumn{6}{|c|}{ S. D. = standard deviation of a single observation. } \\
\hline
\end{tabular}

Tablo 1 - Differences BETWEen MaCRoseismiC Magnitudes COMPUTED FroM EQS. [10], [12], [13] AND [14] AND INSTRUMENTAL MagNitudes M* assigned by Gutenberg, B. and C. F. Richter (GR), M. BAth (B), AND V. KARNIK (K).

Table 1 gives the results of the computations of the differences between macroseismic and instrumental magnitudes. The macroseismic magnitudes were computed from equations [10], [12], [13] and [14], respectively. 
The magnitudes computed from Eq. [10] are given in Table 4. Variations with $M^{*}$ of differences between macroseismic and instrumental magnitudes and percentage frequency distributions of the various magnitude differences are shown in Figures 1,2 and 3, respectively.

Table 1 shows that the standard deviations in all cases but one are around \pm 0.4 to \pm 0.5 . The larger standard deviation of a single observation found from the $\boldsymbol{M}$-determinations by V. Karnik are partly due to large discrepancies (sometimes amounting to one magnitude unit) in the $M$-determinations at Praha (see examples of large discrepancies in Table 4 for earthquakes No. 48, 88, 112), and to the very pronounced tendency of Praha to overestimate the magnitudes for shocks above 6 and underestimate the magnitudes for shocks below 6 . This tendency is clearly indicated (see Fig. 4 and Table 2 ) by the linear relation existing between $\Theta(=\log A+\log I o)$ and $M^{*}(K)$, and the smaller standard deviation of a single observation for $\Theta-M^{*}(K)$.

Table 2 - DifFerences Between Macroseismic Magnitudes COMPUTED FROM EQ. [15] AND Instrumental MagnitUdes $M^{*}$ assigned BY Gutenberg, B. and C. F. Richter (GR), M. B\&til (B) and V. Karnik (K). THE LAST LINE CORRESPONDS TO $\Theta-M^{*}(\mathrm{~K})$.

\begin{tabular}{|c|c|c|c|c|c|}
\hline Magnitude & $h$ & $N$ & $\delta$ & S. E. & S. D. \\
\hline$M^{*}(\mathrm{GR}, \mathrm{B}, \mathrm{K})$ & $n, \quad(n),>n$ & 124 & -0.01 & \pm 0.03 & \pm 0.36 \\
\hline$M^{*}(\mathrm{GR})$ & $n, \quad(n),>n$ & 61 & -0.01 & \pm 0.05 & \pm 0.39 \\
\hline$M^{*}(\mathrm{~B})$ & $n,>n$ & 58 & -0.07 & \pm 0.05 & \pm 0.37 \\
\hline$M^{*}(\mathrm{~K})$ & $n, \quad(n),>n$ & 75 & +0.03 & \pm 0.05 & \pm 0.47 \\
\hline$M=\Theta$ & $n, \quad(n),>n$ & 75 & +0.02 & \pm 0.06 & \pm 0.48 \\
\hline
\end{tabular}

If we apply the very simple formula

$$
M=\Theta+0.2(\Theta-6)
$$

the corresponding standard deviations become much smaller (see Table 2 ). This result may be explained by the following reasoning. By adding the term $0.2(\Theta-6)$ to $\Theta$, the slope of the line we get by plotting $\Theta-M^{*}(G R, B, K$,$) as a function of M^{*}(G R, B, K)$ becomes smaller. By adding the term deduced by the least square method to $\Theta$ we minimize the slope of the line, but the standard deviations become relatively greater (see Table 1). This shows that the magnitudes computed from 
Eq. [15] correspond more closely to the magnitudes determined from body waves. This is clearly indicated by the fact that the slope of the line we get by plotting $\Theta-M^{*}(G R)$ or $\Theta-M^{*}(B)$ against $M^{*}(G R)$, i. e. $M^{*}(B)$, mostly based on body waves, is much smaller than that of the line we get by plotting $\Theta-M^{*}(K)$ against $M^{*}(K)$, which is based on surface waves.
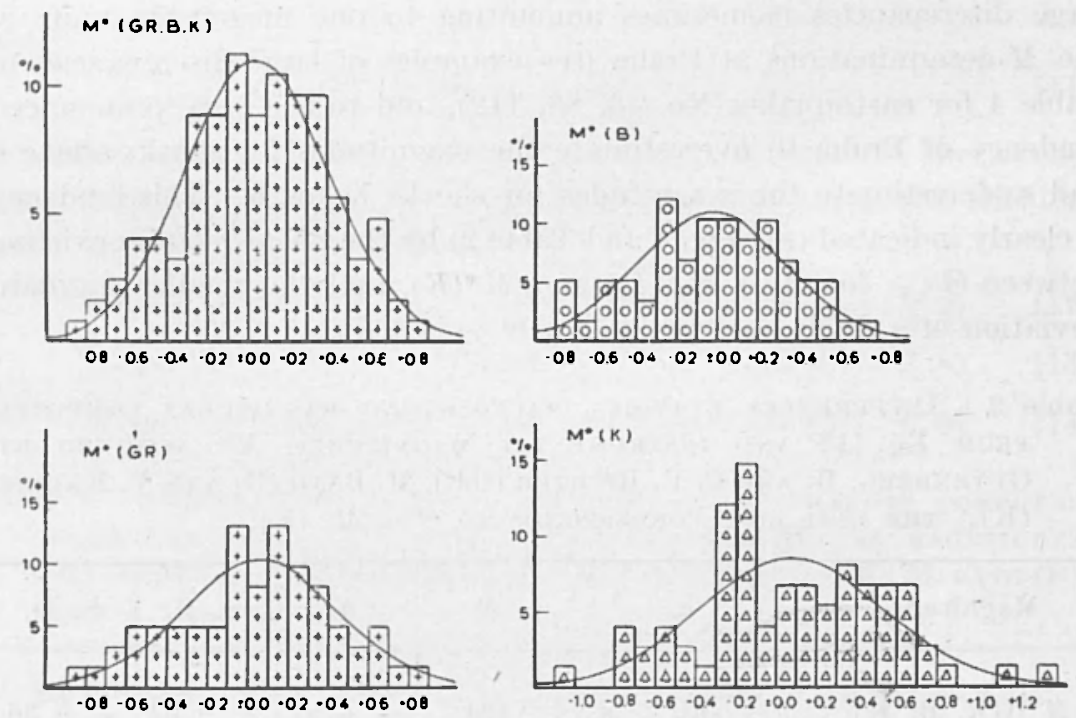

Fig. 5. - Frequency distributions of differences between macroseismic magnitudes computed from Eq. [15] and instrumental magnitudes $M^{*}$ (GR, $\mathrm{B}, \mathrm{K}), M^{*}(\mathrm{GR}), M^{*}(\mathrm{~B})$ and $M^{*}(\mathrm{~K})$.

The percentage frequency distributions of the various magnitude differences which have resulted by applying the formula [15] are shown in Fig. 5. As the limit of an individual $M$-determination from instrumental data is approx. $\pm \mathbf{0 . 3}$, the accuracy of determinino earthquake magnitudes from macroseismic data by the proposed method is very satisfactory. An error of $100 \%$ in the assignment either of the epicentral intensity or of the area of perceptibility corresponds to 0.3 units of the magnitude scale.

b) From Californian Shocks. - In view of the relative reliability of the instrumental and macroseismic data available for shocks in Greece, an attempt was made to apply the proposed method for the $M$-determi- 
nation of California shocks. Data used (see Table 5) were the very reliable elements of California shocks taken from Tables 12 and 15 of the first and second papers on "Earthquake Magnitude, Intensity, Energy, and Acceleration" by B. Gutenberg and C. F. Richter (1942, 1956a).
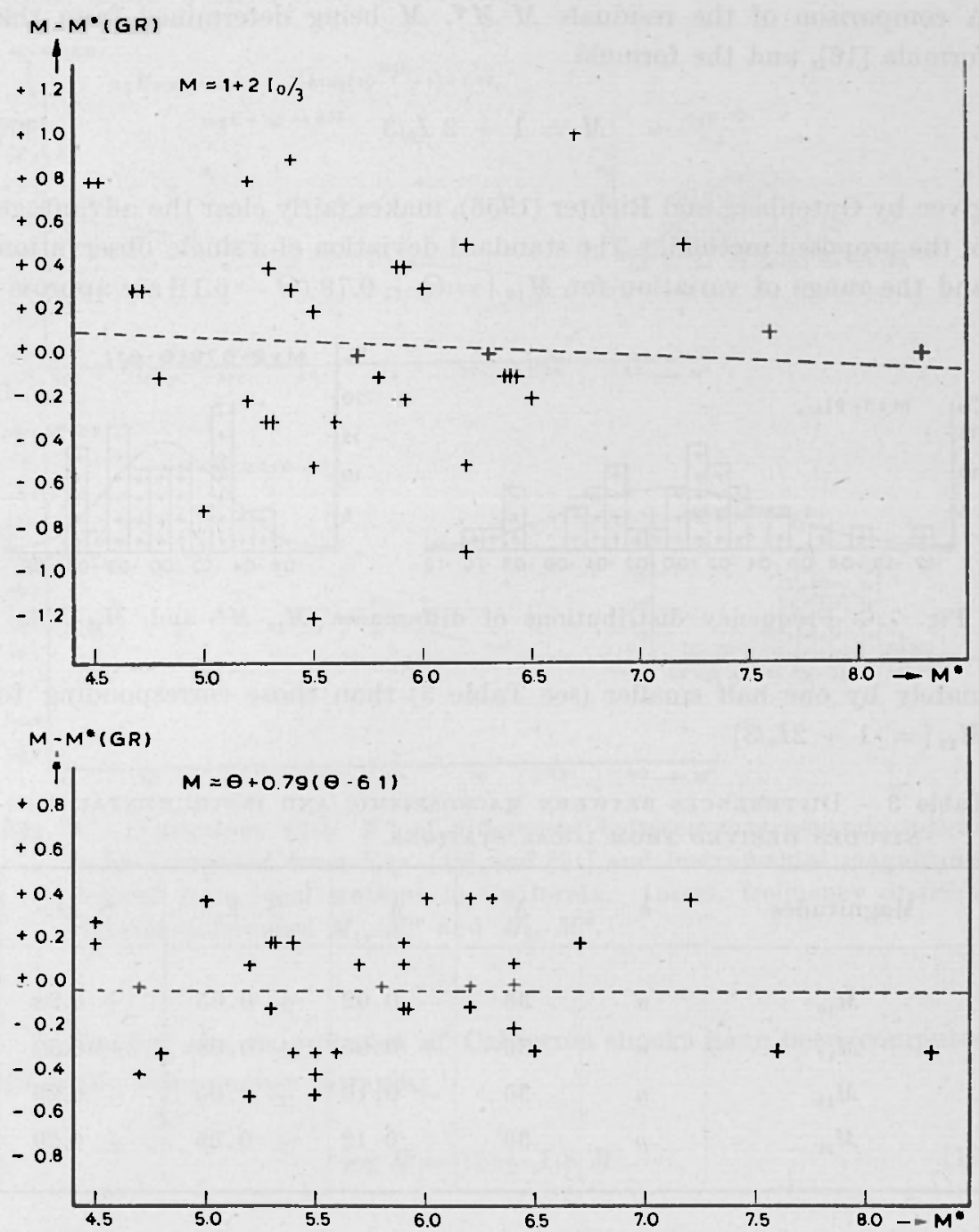

Fig. 6. - Variations with $M^{*}$ of differences between macroseismic magnitudes computed from Eqs. [17] and [16], and instrumental magnitudes derived from local stations in California. 
As shown in Figures 6 and 7 , the macroseismic data of California shocks used for $M$-determination are much better represented by the equation:

$$
M=1.795 \Theta-4.863 \cong \Theta+0.79(\Theta-6.1)
$$

A comparison of the residuals $M-M^{*}, M$ being determined from the formula [16], and the formula

$$
M=1+2 I_{0} / 3
$$

given by Gutenberg and Richter (1956), makes fairly clear the advantage of the proposed method. The standard deviation of a single observation and the range of variation for $M_{16}[=\Theta+0.79(\Theta-6.1)]$ are approxi-
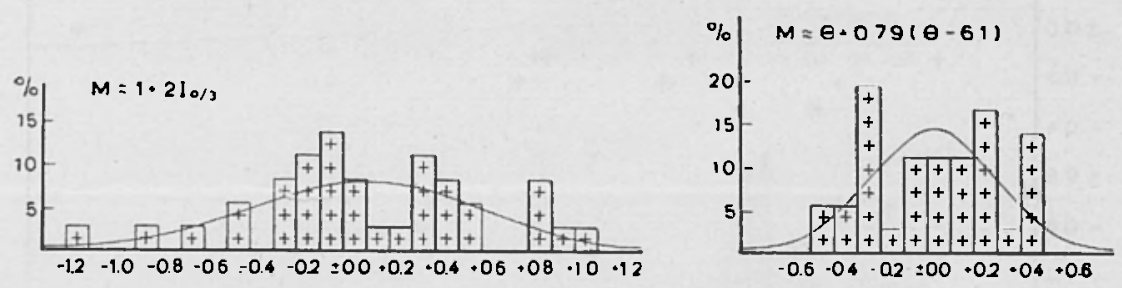

Fig. 7. - Frequency distributions of differences $M_{17}-M^{*}$ and $M_{18}-M^{*}$.

mately by one half smaller (see Table 3 ) than those corresponding to $M_{17}\left[=1+2 I_{0} / 3\right]$

Table 3 - Differences Between MaCroseismic and Instrumental Mag-

\begin{tabular}{|c|c|c|c|c|c|}
\hline Magnitudes & $h$ & $N$ & $\delta$ & S. E. & S. D. \\
\hline$M_{16}$ & $n$ & 36 & -0.02 & \pm 0.05 & \pm 0.28 \\
\hline$M_{17}$ & $n$ & 36 & +0.05 & \pm 0.08 & \pm 0.50 \\
\hline$M_{19}$ & $n$ & 36 & --0.16 & \pm 0.05 & \pm 0.29 \\
\hline.$H_{21}$ & $n$ & 36 & +0.12 & \pm 0.05 & \pm 0.29 \\
\hline
\end{tabular}
NITUDES DERIVED FRON LOCAL STATIONS.

From the equation

$$
M_{L}-M_{B}=04\left(M_{B}-6\right)
$$


found by Gutenberg and Richter (1956a), it is seen that the formula [16] gives approximately the magnitude $M_{L}$, derived from local stations, and that $\Theta$ is nearly equal to the $M_{B}$ determined from body waves. It is therefore confirmed independently that the magnitudes above 6 which were determined from local stations have been overestimated, while those below 6 have been underestimated.
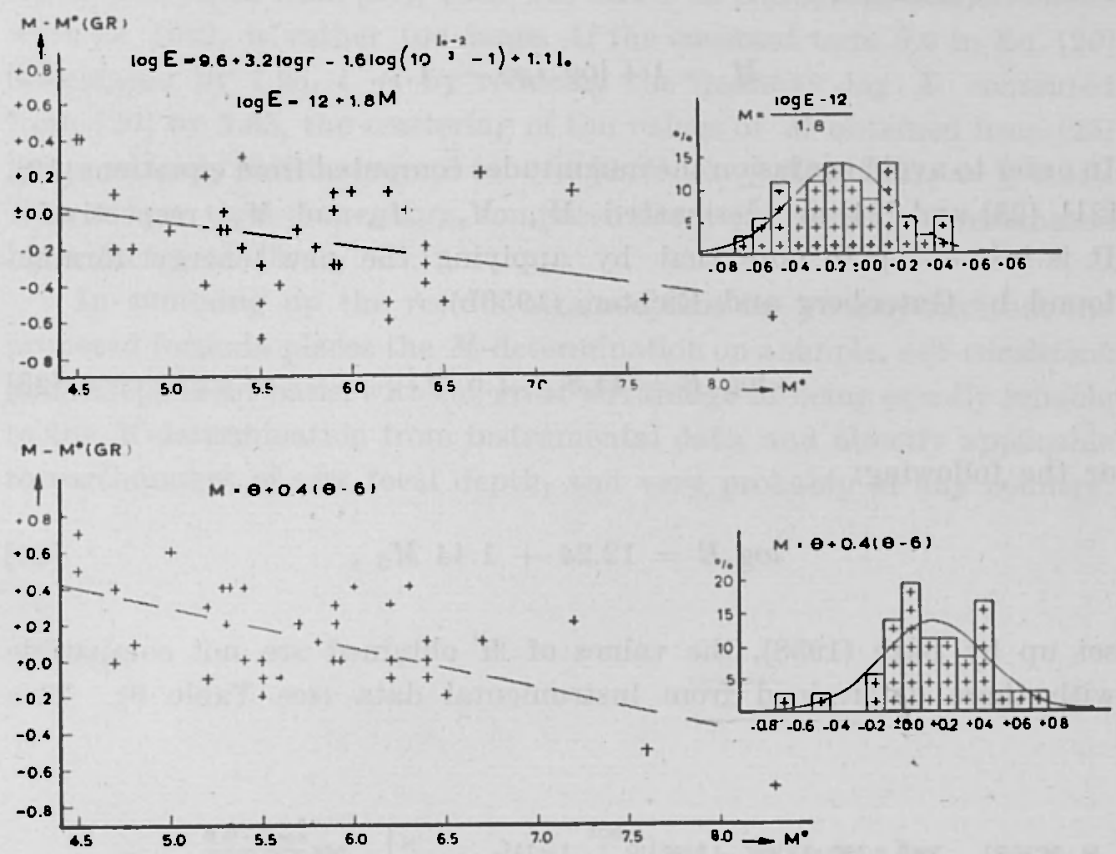

Fig. 8. - Variations with $M^{*}$ of differences between macroseismic magnitudes computed from Eqs. [19] and [21] and instrumental magnitudes derived from local stations in California. Insert, frequency distributions of differences $M_{10^{-}} M^{*}$ and $M_{21} M^{*}$.

Finally, the magnitudes of California shocks have been computed from the older energy formula:

$$
\log E=12+1.8 M
$$

The quantity $\log E$ has been computed from the equation:

$$
\log E=9.6+3.2 \log r-1.6 \log \left(10^{\frac{I_{0}-2}{3}}-1\right)+1.1 I_{0}
$$


found by M. Båth (1953). As we see from Table 3 and Fig. 8, the values of $M$ obtained from [19] and [20] are equally reliable as those found by using the very simple formula:

$$
M=\Theta+0.4(\Theta-6)
$$

or the equivalent:

$$
M=1.4 \log I_{0} r^{2}-1.7
$$

In order to avoid confusion the magnitudes computed from equations [19], [21], [23] and [24] are designated $M_{19}, M_{21}, M_{23}$ and $M_{24}$, respectively. It is interesting to note that by applying the new energy formula found by Gutenberg and Richter, (1956b):

$$
\log E=11.8+1.5 M_{S}
$$

or the following:

$$
\log E=12.24+1.44 M_{S}
$$

set up by Båth (1958), the values of $M$ obtained are not compatible with those determined from instrumental data (see Table 6). This

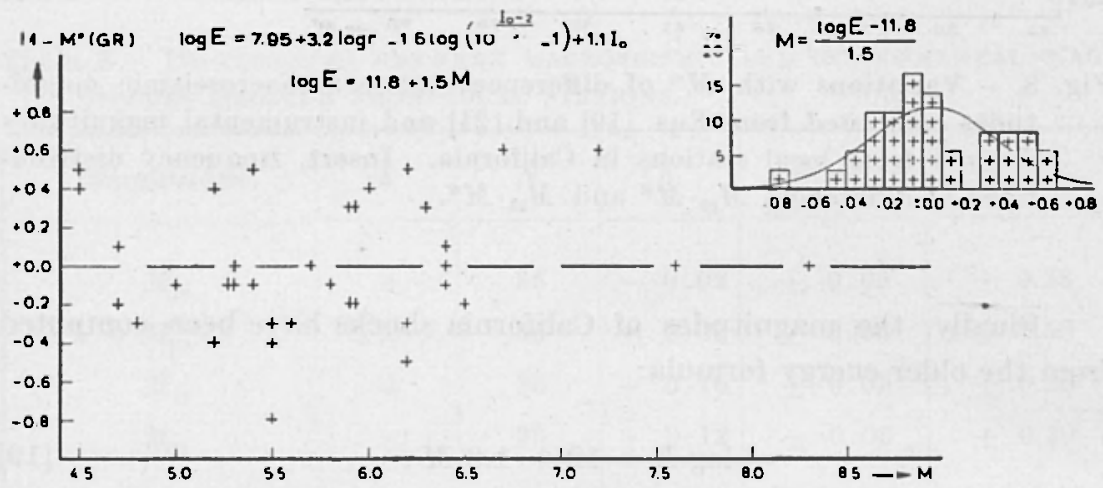

Fig. 9. - Variations with $M^{*}$ of differences between macroseismic magnitudes computed from Eq. [23] and instrumental magnitudes derived from local stations in California. Insert, frequency distributions of differences $M_{23}-M^{*}$. 
means, either the quantity $\log E$ computed from equation [20] is probably too large, or the factor of $M_{S}$ in the new energy formula is too small.

Since the equations [23] and [24] agree very well with each other, although they were derived independently by different methods and by different material, it appears reasonably certain that $\log E$ computed from [20], wich was based on Gutenberg and Richter's work of 1942 , is rather too large. If the constant term 9.6 in Eq. [20] is replaced by 7.95 , i. e. by reducing the quantity $\log E$ computed from [20] by 1.65 , the scattering of the values of $M$ obtained from [23] becomes fairly satisfactory $(\delta=+0.01$, S. E. \pm 0.06, S. D. $= \pm 0.34$ ). This means that the energy computed from [20] was overestimated by a factor of 45 .

In summing up the results attained thus far we may say that the proposed formula places the $M$-determination on a simple, self-consistent and independent basis, with the great advantage of being equally reliable to the $M$-determination from instrumental data, and directly applicable to earthquakes of any focal depth, and very probably of any country. 


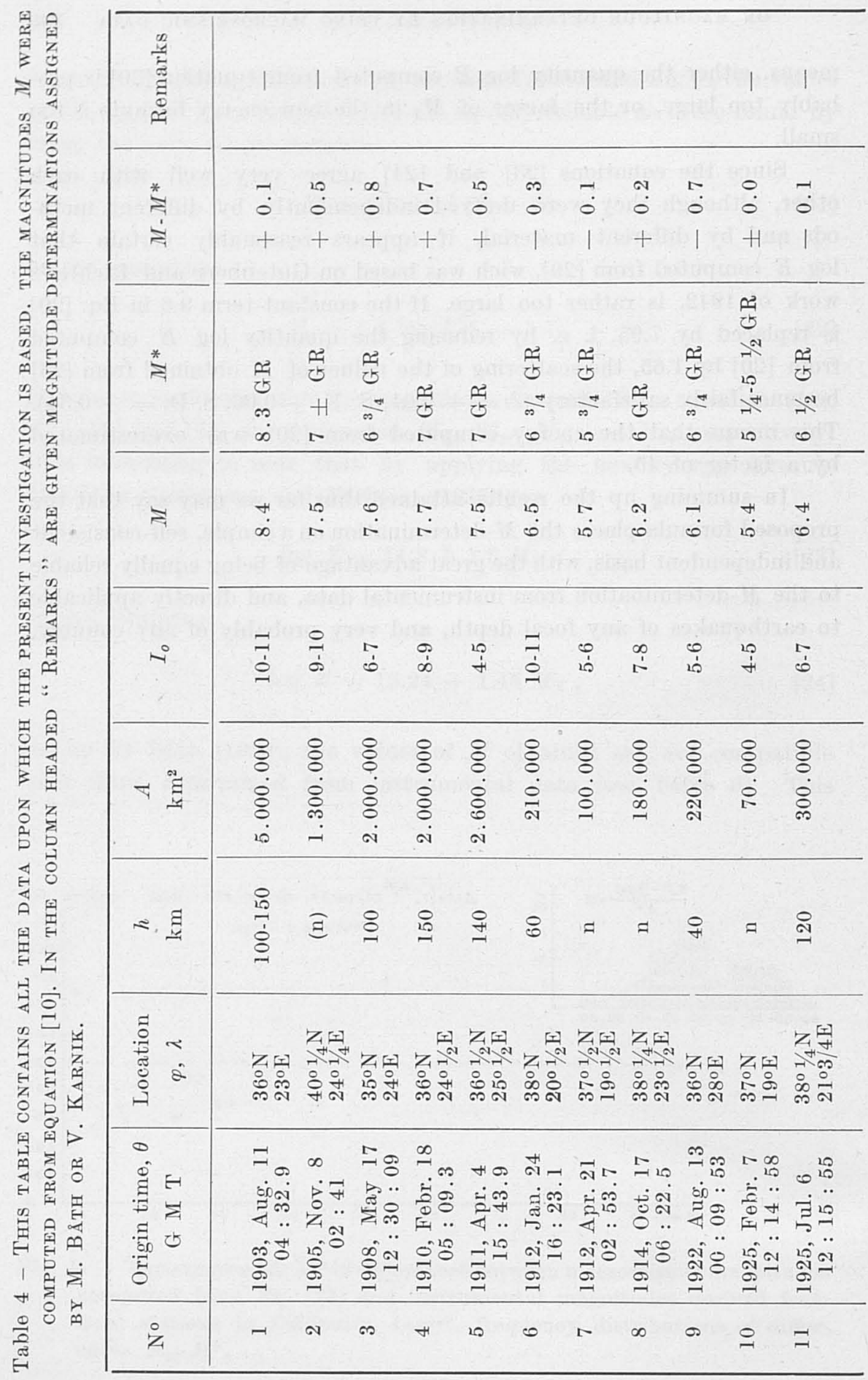




\begin{tabular}{|c|c|c|c|c|c|c|c|c|c|c|c|c|c|}
\hline 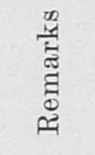 & 1 & 1 & 1 & 1 & 1 & $\begin{array}{l}4 \\
0\end{array}$ & 1 & 1 & 1 & 1 & $\begin{array}{l}\text { जै } \\
\dot{0}\end{array}$ & 1 & 1 \\
\hline $\begin{array}{l}\stackrel{*}{z} \\
\dot{z}\end{array}$ & $\begin{array}{l}10 \\
\dot{0} \\
+\end{array}$ & $\begin{array}{l}10 \\
\dot{0} \\
+\end{array}$ & $\begin{array}{l}0 \\
\dot{0} \\
+\end{array}$ & $\begin{array}{l}0 \\
- \\
+\end{array}$ & $\begin{array}{l}-1 \\
\dot{0} \\
+\end{array}$ & $\begin{array}{l}r \\
0 \\
+\end{array}$ & $\begin{array}{l}N \\
0 \\
1\end{array}$ & $\begin{array}{l}? \\
\dot{0} \\
+\end{array}$ & $\begin{array}{l}\dddot{0} \\
+\end{array}$ & $\begin{array}{l}\dot{H} \\
\dot{0} \\
+\end{array}$ & $\begin{array}{l}\overrightarrow{0} \\
+\end{array}$ & $\begin{array}{l}+1 \\
+ \\
+\end{array}$ & $\begin{array}{l}\stackrel{9}{0} \\
+\end{array}$ \\
\hline$\stackrel{*}{\rightrightarrows}$ & 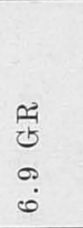 & 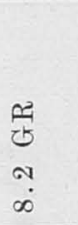 & 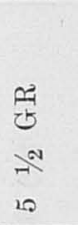 & $\begin{array}{l}\stackrel{2}{2} \\
\stackrel{1}{0} \\
0 \\
\end{array}$ & $\begin{array}{l}\stackrel{2}{2} \\
0 \\
0 \\
0\end{array}$ & $\begin{array}{l}\stackrel{c}{J} \\
\frac{+1}{\infty} \\
0\end{array}$ & $\begin{array}{l}1 \\
0 \\
0\end{array}$ & $\underset{0}{0}$ & $\begin{array}{l}1 \\
0 \\
\text { is }\end{array}$ & 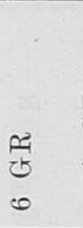 & 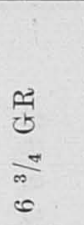 & 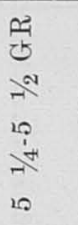 & 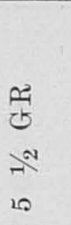 \\
\hline$z$ & $\stackrel{+}{\therefore}$ & $\stackrel{1}{\infty}$ & $\because$ & $\stackrel{0}{\infty}$ & $\stackrel{0}{N}$ & $\stackrel{?}{2}$ & is & $?$ & is. & H. & 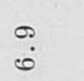 & $\infty$ & is \\
\hline -i & $\begin{array}{l}\infty \\
1 \\
1\end{array}$ & $\vec{\sigma}$ & $\stackrel{10}{4}$ & $\stackrel{\infty}{i}$ & $\frac{9}{1}$ & $=$ & $\stackrel{\infty}{i}$ & $\stackrel{\circ}{\circ}$ & $\dot{\infty}$ & $\stackrel{\infty}{i}$ & $\frac{0}{0}$ & t) & ف̊ \\
\hline ૪ है हี & $\begin{array}{l}8 \\
8 \\
\dot{8} \\
\dot{0} \\
\dot{-1}\end{array}$ & $\begin{array}{l}\delta \\
8 \\
8 \\
8 \\
\infty\end{array}$ & $\begin{array}{l}8 \\
8 \\
0 \\
\text { ㅇํ }\end{array}$ & $\begin{array}{l}8 \\
\delta \\
\dot{8} \\
\dot{0} \\
\dot{0}\end{array}$ & $\begin{array}{l}8 \\
8 \\
\dot{8} \\
10\end{array}$ & \begin{tabular}{l}
$\stackrel{8}{8}$ \\
$\dot{8}$ \\
$\stackrel{\circ}{+}$ \\
\hdashline
\end{tabular} & $\begin{array}{l}8 \\
8 \\
\dot{8}\end{array}$ & $\begin{array}{l}8 \\
\dot{8} \\
\dot{8}\end{array}$ & $\begin{array}{l}8 \\
\dot{8} \\
\dot{8}\end{array}$ & $\begin{array}{l}8 \\
8 \\
0 \\
0 \\
\text { คे }\end{array}$ & $\begin{array}{l}8 \\
8 \\
0 \\
0 \\
+1\end{array}$ & \begin{tabular}{l}
8 \\
8 \\
8 \\
\hdashline
\end{tabular} & $\begin{array}{l}8 \\
8 \\
8\end{array}$ \\
\hline$\approx E$ & छ & 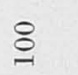 & 足 & 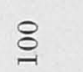 & 요 & $\stackrel{\circ}{-1}$ & $\Rightarrow$ & $\Rightarrow$ & $\Rightarrow$ & $\Rightarrow$ & $\Rightarrow$ & $\Rightarrow$ & $\stackrel{8}{8}$ \\
\hline 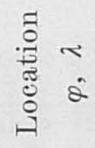 & 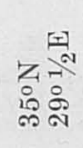 & 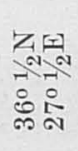 & 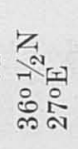 & 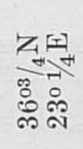 & 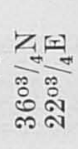 & 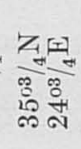 & 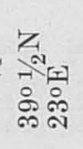 & 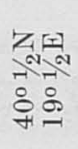 & $\begin{array}{l}751 \\
1010 \\
80 \\
00 \\
+9\end{array}$ & 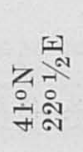 & 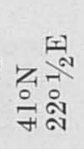 & 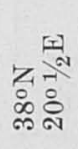 & 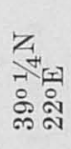 \\
\hline 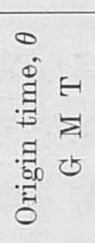 & 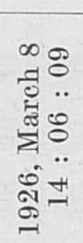 & 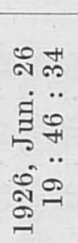 & 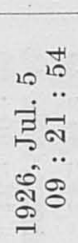 & 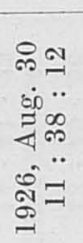 & 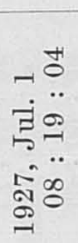 & 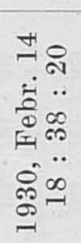 & 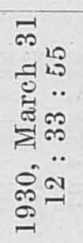 & 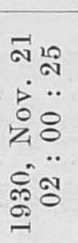 & 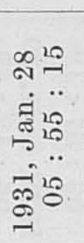 & 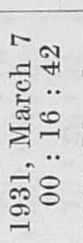 & 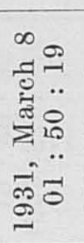 & 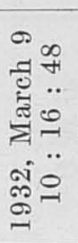 & 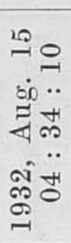 \\
\hline 艺 & $\stackrel{\sim}{\sim}$ & $\dddot{-1}$ & 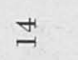 & 20 & $\stackrel{\bullet}{ }$ & 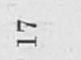 & $\stackrel{\infty}{\sim}$ & $\stackrel{\theta}{-}$ & ฉิ & $\vec{\sim}$ & สี & ลึ & ने \\
\hline
\end{tabular}




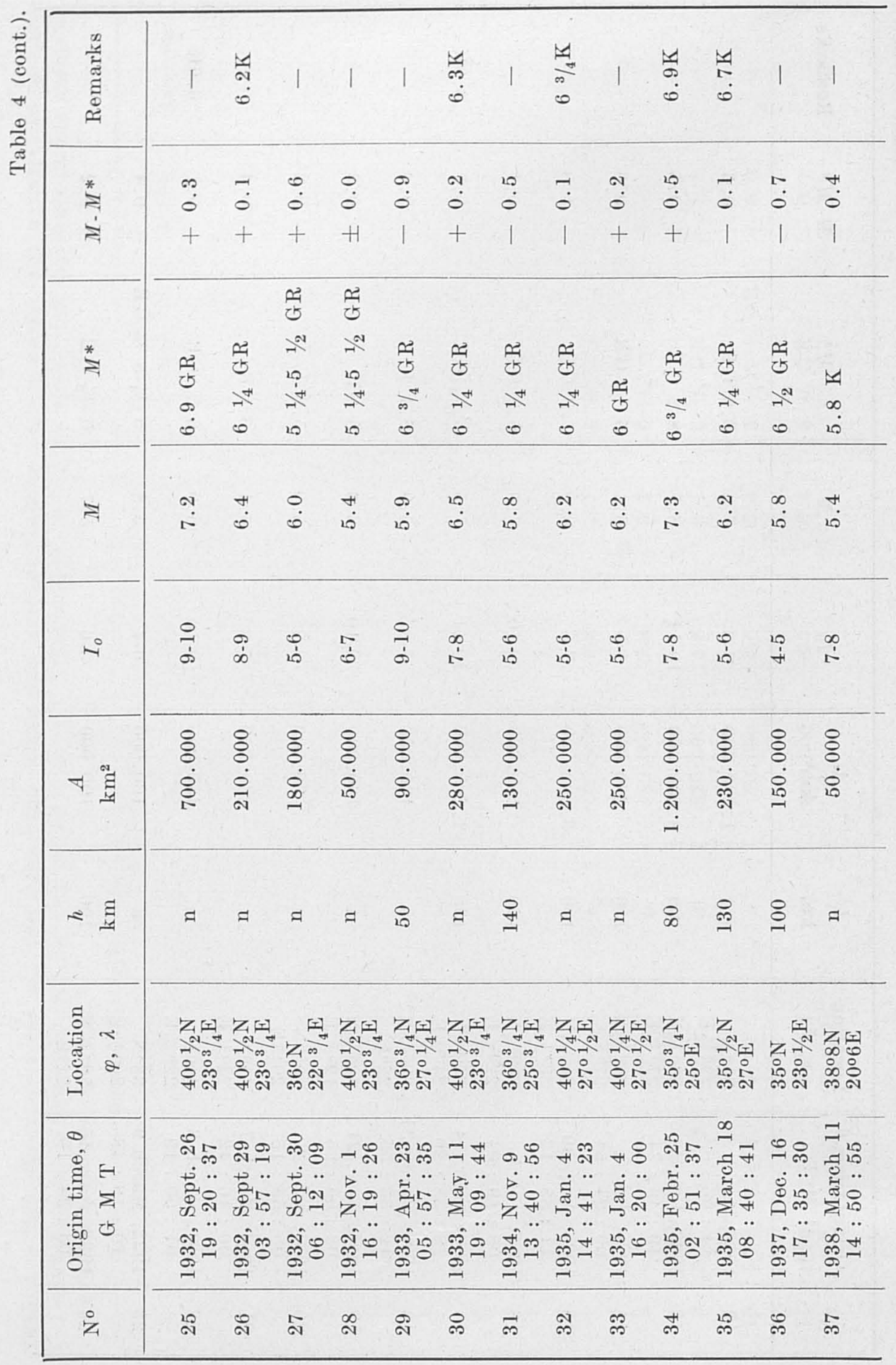




\begin{tabular}{|c|c|c|c|c|c|c|c|c|c|c|c|c|c|}
\hline 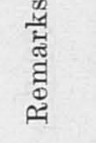 & 1 & $\begin{array}{l}\stackrel{4}{A} \\
\stackrel{0}{0}\end{array}$ & 1 & $\begin{array}{l}M \\
10\end{array}$ & $\begin{array}{l}\qquad 1 \\
0 \\
\dot{0}\end{array}$ & औै & $\begin{array}{l}\text { y. } \\
\text { is }\end{array}$ & $\frac{\not}{5}$ & मै & 1 & $\begin{array}{l}\text { है } \\
\text { r }\end{array}$ & 1 & $\frac{1}{10}$ \\
\hline $\begin{array}{l}\stackrel{*}{\exists} \\
\dot{y}\end{array}$ & $\begin{array}{r}\dddot{0} \\
1\end{array}$ & $\begin{array}{l}? \\
0 \\
+\end{array}$ & $\begin{array}{l}\infty \\
\dot{0} \\
1\end{array}$ & $\begin{array}{l}\stackrel{9}{0} \\
+\end{array}$ & $\begin{array}{l}0 \\
\dot{0} \\
H\end{array}$ & $\begin{array}{l}\dddot{0} \\
0 \\
1\end{array}$ & $\begin{array}{l}? \\
0 \\
1\end{array}$ & $\begin{array}{l}0 \\
\dot{0} \\
H\end{array}$ & $\begin{array}{l}0 \\
0 \\
+\end{array}$ & $\begin{array}{l}m \\
0 \\
1\end{array}$ & $\begin{array}{l}7 \\
1\end{array}$ & $\begin{array}{l}\overrightarrow{0} \\
H\end{array}$ & $\begin{array}{l}0 \\
\dot{0} \\
H\end{array}$ \\
\hline$\stackrel{*}{7}$ & 19 & $\begin{array}{l}\frac{0}{21} \\
\text { चु } \\
\frac{-1}{10} \\
20\end{array}$ & 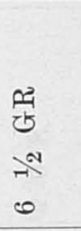 & 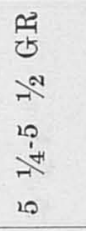 & $\begin{array}{l}\frac{12}{d 5} \\
\lambda^{2} \\
0\end{array}$ & $\begin{array}{l}0 \\
0 \\
0 \\
0 \\
0\end{array}$ & $\begin{array}{l}2 \\
0 \\
0 \\
0\end{array}$ & $\begin{array}{l}2 \\
\text { 2ै } \\
0 \\
0\end{array}$ & $\begin{array}{l}\frac{0}{5} \\
\lambda^{2} \\
0 \\
0\end{array}$ & $\begin{array}{ll}4 \\
20\end{array}$ & $\begin{array}{l}27 \\
0 \\
0\end{array}$ & $\begin{array}{l}\text { चु } \\
H \\
+10 \\
-10\end{array}$ & $\begin{array}{l}\text { चु } \\
\text { [- }\end{array}$ \\
\hline$\vec{z}$ & 19. & $\ddot{0}$ & is & $\stackrel{\varphi}{10}$ & ?? & $\begin{array}{l}0 \\
0\end{array}$ & 10 & $\stackrel{0}{0}$ & $\stackrel{\oplus}{\mathfrak{D}}$ & $\stackrel{0}{10}$ & is & $\stackrel{0}{10}$ & $\stackrel{0}{i}$ \\
\hline -i & $\stackrel{\infty}{i}$ & $\stackrel{\infty}{\vdots}$ & فُ & is & $\begin{array}{l}0 \\
\dot{b}\end{array}$ & $\underset{b}{\dot{b}}$ & $\stackrel{\infty}{\check{L}}$ & b & के & $\stackrel{10}{+}$ & ip & ig & $\begin{array}{l}\dot{b} \\
\infty\end{array}$ \\
\hline 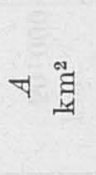 & $\begin{array}{l}8 \\
8 \\
0 \\
10\end{array}$ & $\begin{array}{l}8 \\
8 \\
0 \\
10 \\
-1\end{array}$ & $\begin{array}{l}8 \\
\stackrel{8}{0} \\
\stackrel{0}{0}\end{array}$ & $\begin{array}{l}8 \\
8 \\
\dot{8}\end{array}$ & $\begin{array}{l}8 \\
8 \\
0 \\
\stackrel{8}{\circ} \\
\text { ค. }\end{array}$ & 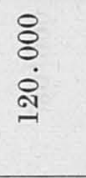 & $\begin{array}{l}8 \\
: \\
\dot{0}\end{array}$ & $\begin{array}{l}8 \\
\vdots \\
\dot{0} \\
-1\end{array}$ & $\begin{array}{l}8 \\
8 \\
\dot{8} \\
\infty \\
\dot{-1}\end{array}$ & $\begin{array}{l}8 \\
0 \\
\dot{9}\end{array}$ & $\begin{array}{l}8 \\
8 \\
0 \\
\infty \\
-1\end{array}$ & $\begin{array}{l}8 \\
8 \\
0 \\
8\end{array}$ & $\begin{array}{l}8 \\
8 \\
0 \\
0 \\
10\end{array}$ \\
\hline$\therefore$ घี & $\Rightarrow$ & $\Rightarrow$ & $\stackrel{8}{\circ}$ & $\Rightarrow$ & $\Rightarrow$ & $\Rightarrow$ & $\Rightarrow$ & $\stackrel{8}{8}$ & $\stackrel{\circ}{=}$ & $\Rightarrow$ & $\stackrel{\infty}{\infty}$ & $\begin{array}{l}H \\
8\end{array}$ & $\stackrel{\infty}{\sim}$ \\
\hline 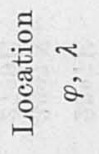 & 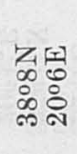 & 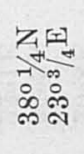 & 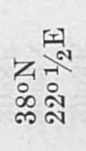 & 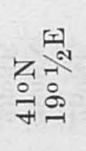 & 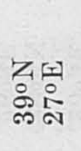 & 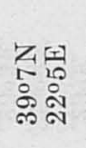 & 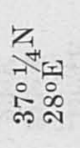 & 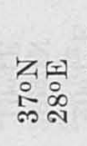 & 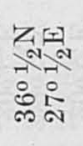 & 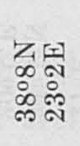 & 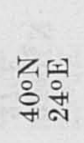 & 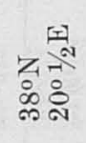 & 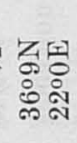 \\
\hline 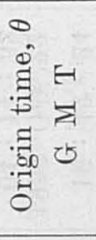 & 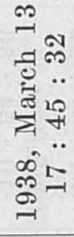 & 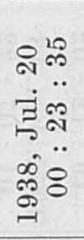 & 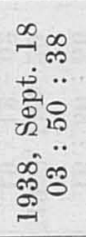 & 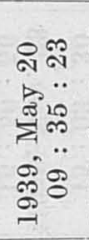 & 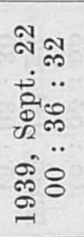 & 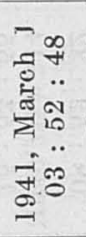 & 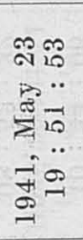 & 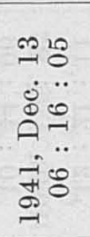 & 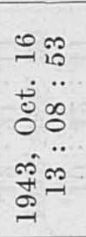 & 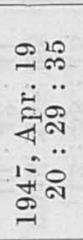 & 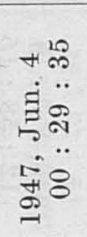 & 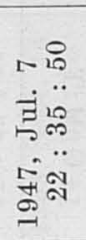 & 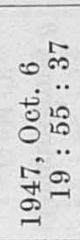 \\
\hline$\dot{z}$ & 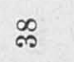 & శ్లి & of & $F$ & Fี & $\stackrel{\Re}{F}$ & $\not$ & $\stackrel{10}{7}$ & $\stackrel{f}{\not}$ & 담 & $\stackrel{\infty}{+}$ & $\stackrel{P}{+}$ & is \\
\hline
\end{tabular}




\begin{tabular}{|c|c|c|c|c|c|c|c|c|c|c|c|c|c|}
\hline 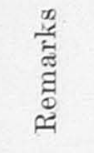 & $\frac{\mathscr{H}}{\stackrel{5}{\sim}}$ & $\begin{array}{l}1 \\
\infty \\
0\end{array}$ & $\frac{1}{10}$ & 1 & 1 & 1 & $\frac{1}{0}$ & 1 & 1 & $\stackrel{4}{40}$ & $\begin{array}{l}\text { 늠 } \\
10\end{array}$ & $\begin{array}{l}10 \\
10\end{array}$ & $\begin{array}{l}\text { है } \\
\text { 10 }\end{array}$ \\
\hline $\begin{array}{l}\stackrel{*}{\sharp} \\
\stackrel{4}{\sharp}\end{array}$ & $\begin{array}{l}0 \\
\dot{0} \\
1\end{array}$ & $\begin{array}{l}\because \\
\dot{0} \\
+\end{array}$ & $\begin{array}{l}\dddot{0} \\
+\end{array}$ & $\begin{array}{l}\dddot{0} \\
1\end{array}$ & $\begin{array}{l}0 \\
\dot{0} \\
+\end{array}$ & $\begin{array}{l}+1 \\
\dot{0} \\
1\end{array}$ & $\begin{array}{l}0 \\
\dot{0} \\
1\end{array}$ & $\begin{array}{l}0 \\
\dot{0} \\
H\end{array}$ & $\begin{array}{l}? \\
0 \\
1\end{array}$ & $\begin{array}{l}0 \\
\dot{0} \\
H\end{array}$ & $\begin{array}{l}9 \\
0 \\
1\end{array}$ & $\begin{array}{l}\overrightarrow{0} \\
+\end{array}$ & $\begin{array}{l}? \\
+\end{array}$ \\
\hline$\stackrel{*}{z}$ & 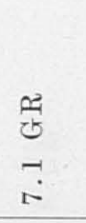 & 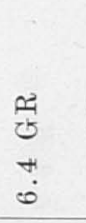 & 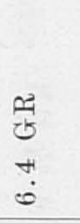 & $\begin{array}{l}15 \\
4 \\
10\end{array}$ & $\begin{array}{l}19 \\
10 \\
0\end{array}$ & $\begin{array}{l}15 \\
15\end{array}$ & 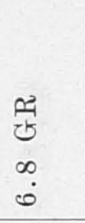 & $\begin{array}{l}15 \\
10\end{array}$ & $\begin{array}{l}11 \\
0 \\
10\end{array}$ & 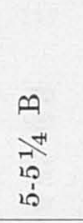 & $\frac{\sqrt{10}}{\pi^{\infty}}$ & 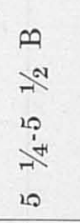 & $\stackrel{10}{10}$ \\
\hline$\Rightarrow$ & ?9 & $\stackrel{10}{0}$ & $\stackrel{10}{0}$ & 70 & $\stackrel{\square}{\therefore}$ & 20 & $\stackrel{\oplus}{0}$ & 70 & $\begin{array}{l}\infty \\
\dot{+}\end{array}$ & 7 & मे & 10. & 10 \\
\hline$-i$ & $\dot{a}_{i}$ & $\stackrel{\circ}{\stackrel{1}{\sigma}}$ & $\stackrel{\circ}{\circ}$ & $\stackrel{20}{4}$ & $\stackrel{10}{4}$ & मे & $\dot{\infty}$ & ஹீ & id & $\stackrel{\infty}{\stackrel{\infty}{\perp}}$ & $\frac{20}{4}$ & $\stackrel{\infty}{\stackrel{\infty}{\check{r}}}$ & id \\
\hline$\nabla \stackrel{\text { sa }}{\mathbb{E}}$ & 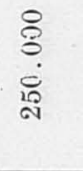 & $\begin{array}{l}8 \\
8 \\
0 \\
0 \\
10 \\
\text { a }\end{array}$ & $\begin{array}{l}8 \\
8 \\
0 \\
10 \\
\text { ง }\end{array}$ & $\begin{array}{c}8 \\
0 \\
10 \\
4\end{array}$ & $\begin{array}{l}8 \\
\dot{0} \\
\dot{8} \\
\stackrel{\circ}{\circ} \\
-1\end{array}$ & $\begin{array}{l}8 \\
8 \\
\infty\end{array}$ & $\begin{array}{l}8 \\
8 \\
\dot{8} \\
1\end{array}$ & $\begin{array}{l}8 \\
8 \\
\dot{0}\end{array}$ & $\begin{array}{l}8 \\
\text { की } \\
\text { iी }\end{array}$ & $\begin{array}{l}8 \\
\dot{8} \\
\dot{0}\end{array}$ & $\begin{array}{l}8 \\
\vdots \\
\dot{0}\end{array}$ & $\begin{array}{l}8 \\
8 \\
0 \\
0\end{array}$ & $\begin{array}{l}8 \\
\dot{8} \\
\dot{0}\end{array}$ \\
\hline$\approx$ I & 아 & $\Rightarrow$ & $\Rightarrow$ & $\Rightarrow$ & $\infty$ & $\Rightarrow$ & $\approx$ & a & $\Rightarrow$ & $\Rightarrow$ & $\Rightarrow$ & $\Rightarrow$ & व \\
\hline 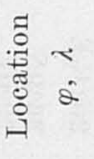 & 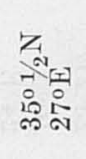 & 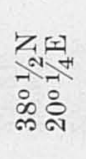 & 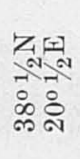 & 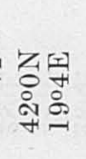 & 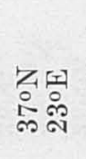 & 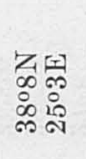 & 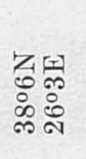 & 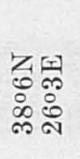 & 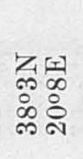 & 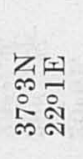 & 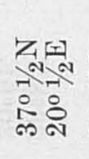 & 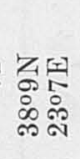 & $\begin{array}{l}\text { Z1 } \\
\text { Z1 } \\
0 \\
0 \\
0 \\
0 \\
0 \\
0\end{array}$ \\
\hline 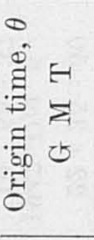 & 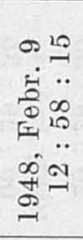 & 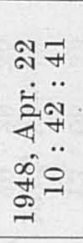 & 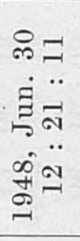 & 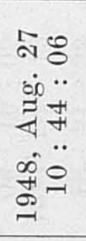 & 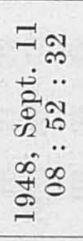 & 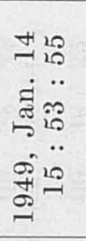 & 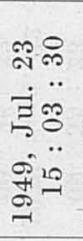 & 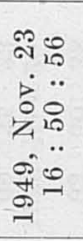 & 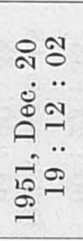 & 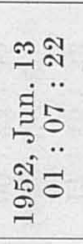 & 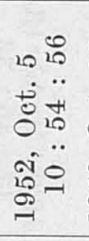 & 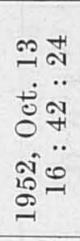 & 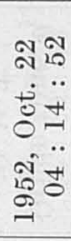 \\
\hline ㅁ & 20 & 임 & is & 20 & 10 & 20 & is & $\stackrel{2}{\llcorner 0}$ & is & تّ & 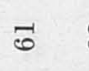 & ชู & ஜ \\
\hline
\end{tabular}




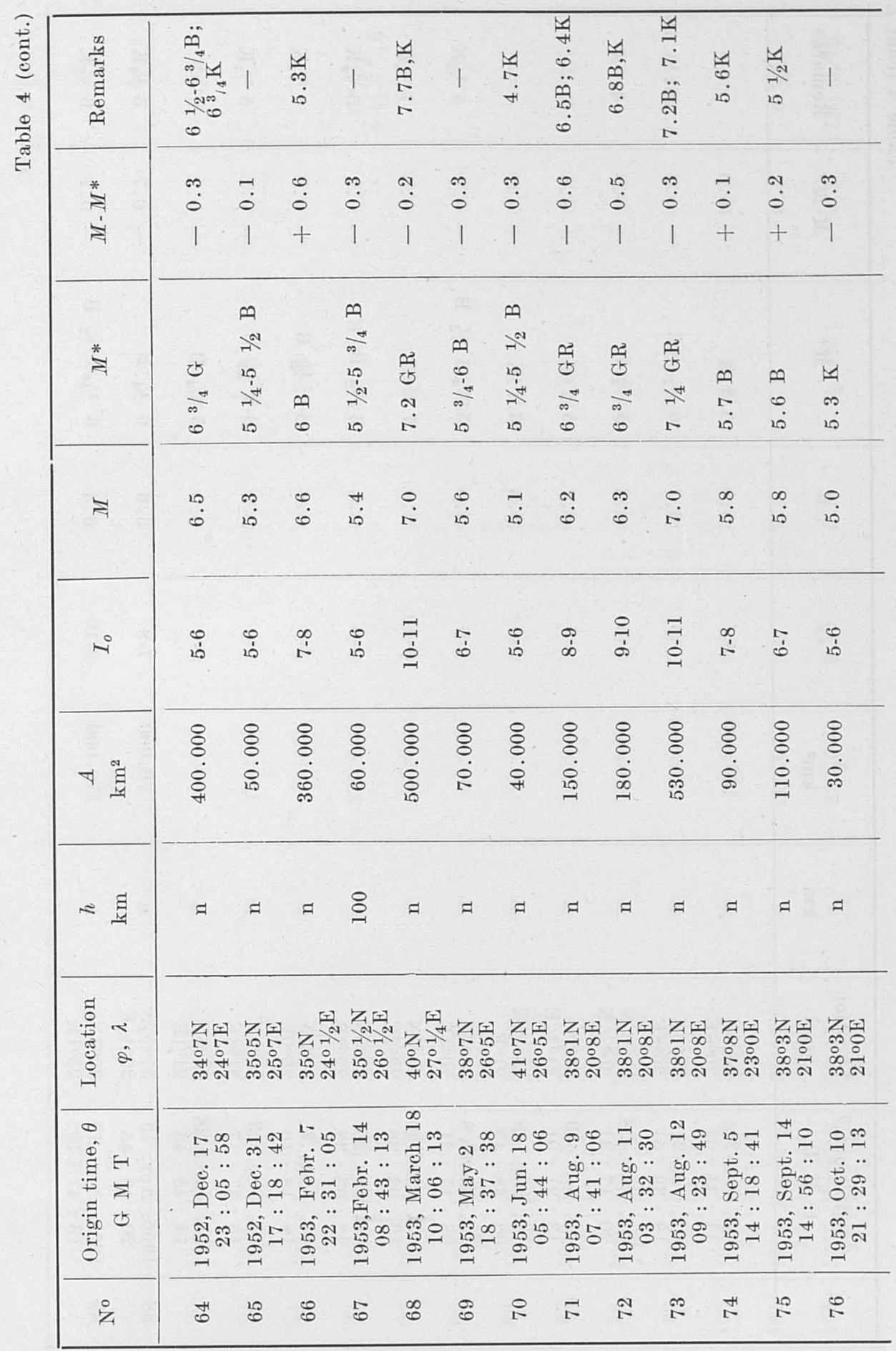




\begin{tabular}{|c|c|c|c|c|c|c|c|c|c|c|c|c|c|}
\hline 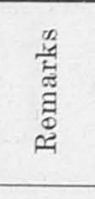 & 1 & $\begin{array}{l}\frac{1}{10} \\
0 \\
\ddot{0} \\
\dot{0} \\
\dot{0}\end{array}$ & $\begin{array}{l}\text { है } \\
\text { मे }\end{array}$ & 1 & 1 & $\frac{11}{10}$ & $\overbrace{}^{\infty}$ & $\frac{11}{10}$ & $\frac{14}{10}$ & $\frac{1}{10}$ & 1 & $\frac{1}{20}$ & $\frac{1}{0}$ \\
\hline $\begin{array}{l}\stackrel{*}{\sharp} \\
\stackrel{\sharp}{\sharp}\end{array}$ & $\begin{array}{l}0 \\
0 \\
+\end{array}$ & $\begin{array}{l}9 \\
\dot{0} \\
1\end{array}$ & $\begin{array}{l}0 \\
\dot{0} \\
H\end{array}$ & $\begin{array}{l}\dot{10} \\
+ \\
+\end{array}$ & $\begin{array}{l}\stackrel{0}{0} \\
+\end{array}$ & $\begin{array}{l}4 \\
0 \\
1\end{array}$ & $\begin{array}{l}4 \\
0\end{array}$ & $\begin{array}{c}-1 \\
1\end{array}$ & $\begin{array}{l}? \\
0 \\
1\end{array}$ & $\begin{array}{l}-1 \\
\dot{0} \\
1\end{array}$ & $\begin{array}{l}? \\
0 \\
1\end{array}$ & $\begin{array}{l}0 \\
1\end{array}$ & $\begin{array}{l}-1 \\
1\end{array}$ \\
\hline$\stackrel{*}{\rightrightarrows}$ & $\begin{array}{l}19 \\
10 \\
10\end{array}$ & 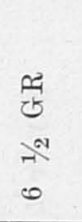 & $\begin{array}{l}n \\
\lambda^{n} \\
10\end{array}$ & $\begin{array}{l}19 \\
10 \\
10\end{array}$ & $\begin{array}{l}11 \\
71 \\
10\end{array}$ & 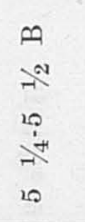 & $\begin{array}{l}\frac{2}{5} \\
1\end{array}$ & $\begin{array}{l}\infty \\
-10 \\
20\end{array}$ & $\begin{array}{c}n \\
\lambda^{+1} \\
0 \\
\dot{b}\end{array}$ & $\begin{array}{l}m \\
\lambda^{+1} \\
0 \\
0 \\
0\end{array}$ & $\begin{array}{l}\infty \\
\frac{\infty}{\infty} \\
20\end{array}$ & $\begin{array}{l}\infty \\
\lambda^{+1} \\
0\end{array}$ & $\begin{array}{c}n \\
\lambda^{N} \\
0 \\
\lambda^{\infty} \\
0\end{array}$ \\
\hline$\nexists$ & is & $?$ & 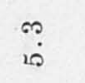 & 5 & $\ddot{10}$ & $\stackrel{0}{10}$ & $\stackrel{0}{0}$ & in & ${ }_{i 0}^{\infty}$ & $\stackrel{0}{0}$ & $\ddot{10}$ & $\begin{array}{l}0 \\
20\end{array}$ & $\dddot{0}$ \\
\hline$-i$ & ț & $\stackrel{\infty}{\stackrel{1}{\leftarrow}}$ & $\stackrel{10}{4}$ & $\begin{array}{l}a_{i} \\
\infty\end{array}$ & $\begin{array}{l}0 \\
10 \\
10\end{array}$ & id & $\stackrel{0}{\circ}$ & $\begin{array}{l}0 \\
1 \\
16\end{array}$ & $\stackrel{2}{4}$ & $\stackrel{\infty}{i}$ & $\stackrel{\infty}{i}$ & $\stackrel{\infty}{\stackrel{1}{1}}$ & $\stackrel{\circ}{\circ}$ \\
\hline$\nabla \stackrel{\text { I }}{\sharp}$ & $\begin{array}{l}8 \\
8 \\
0 \\
\stackrel{1}{9}\end{array}$ & $\begin{array}{l}8 \\
0 \\
\dot{\sigma} \\
\dot{1}\end{array}$ & $\begin{array}{l}8 \\
8 \\
0 \\
0\end{array}$ & $\begin{array}{l}8 \\
8 \\
\dot{0}\end{array}$ & $\begin{array}{l}8 \\
8 \\
0 \\
0\end{array}$ & $\begin{array}{l}8 \\
8 \\
0 \\
0\end{array}$ & $\begin{array}{l}8 \\
8 \\
\dot{\delta} \\
\text { जे }\end{array}$ & $\begin{array}{l}8 \\
8 \\
0\end{array}$ & $\begin{array}{l}8 \\
8 \\
0 \\
10 \\
-1\end{array}$ & $\begin{array}{l}8 \\
8 \\
0 \\
\dot{0} \\
-1\end{array}$ & $\begin{array}{l}8 \\
8 \\
0\end{array}$ & $\begin{array}{l}8 \\
8 \\
0 \\
0\end{array}$ & $\begin{array}{l}8 \\
8 \\
0 \\
0 \\
-1\end{array}$ \\
\hline$\approx$ 可 & $\Rightarrow$ & $\approx$ & $\Rightarrow$ & $\Rightarrow$ & $\Rightarrow$ & $\Rightarrow$ & $\Rightarrow$ & $\Rightarrow$ & $\Rightarrow$ & $\Rightarrow$ & $\Rightarrow$ & $\Rightarrow$ & $\Rightarrow$ \\
\hline : & 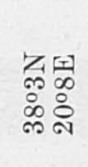 & 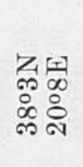 & 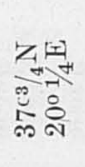 & 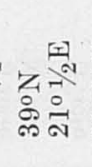 & 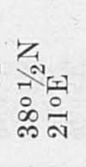 & 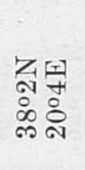 & 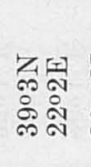 & 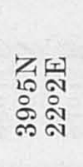 & 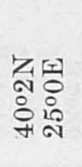 & 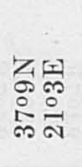 & 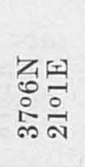 & 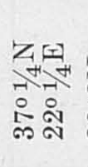 & 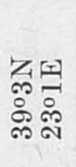 \\
\hline 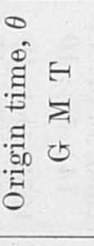 & 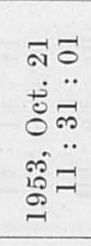 & 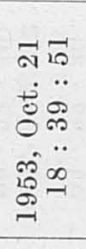 & 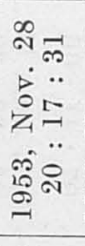 & 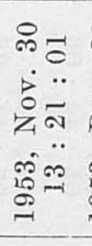 & 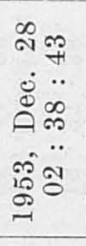 & 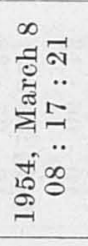 & 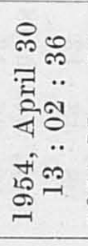 & 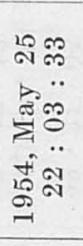 & 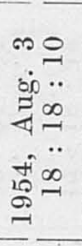 & 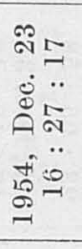 & 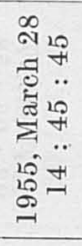 & 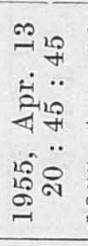 & 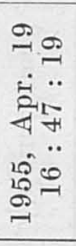 \\
\hline iz & 上 & $\stackrel{\infty}{1}$ & $\stackrel{R}{1}$ & $\infty$ & $\vec{\infty}$ & ๙ & $\mathscr{\infty}$ & 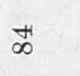 & 10 & $\infty$ & 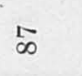 & $\infty$ & $\underset{\infty}{\infty}$ \\
\hline
\end{tabular}




\begin{tabular}{|c|c|c|c|c|c|c|c|c|c|c|c|c|c|}
\hline 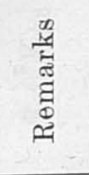 & $\begin{array}{l}y \\
\text { क्ष }\end{array}$ & 1 & 1 & 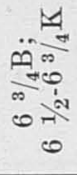 & 1 & & 1 & $\frac{1}{10}$ & 1 & $\Rightarrow$ & 1 & $\frac{N}{0}$ & $\begin{array}{c}0 \\
0 \\
\ddot{0} \\
\ddot{0}\end{array}$ \\
\hline$\stackrel{*}{\stackrel{*}{Z}}$ & $\ddot{0}$ & $\dddot{0}$ & $?$ & i? & $\dot{0}$ & $\ddot{0}$ & $\dot{0}$ & ণ. & ֻ. & $\because$ & ণ & $\ddot{0}$ & $\stackrel{\sim}{0}$ \\
\hline 견 & + & + & + & 1 & 1 & 1 & 1 & + & + & 1 & 1 & 1 & + \\
\hline$\stackrel{*}{*}$ & $\oplus_{0}^{\infty}$ & $\begin{array}{l}\infty 0 \\
\overbrace{}^{+1} \\
00\end{array}$ & 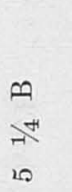 & 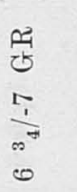 & $\begin{array}{l}\infty 0 \\
-4 \\
10\end{array}$ & $\begin{array}{l}10 \\
10\end{array}$ & $\begin{array}{l}\oplus \\
\stackrel{.}{0}\end{array}$ & $\begin{array}{l}n \\
\stackrel{-}{0}\end{array}$ & $\begin{array}{l}m \\
4 \\
10\end{array}$ & $\frac{\infty}{\infty}$ & $\begin{array}{l}n \\
\pi^{N} \\
10\end{array}$ & $\begin{array}{l}\infty \\
\infty \\
0\end{array}$ & 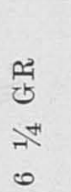 \\
\hline 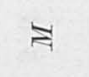 & 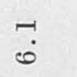 & $\stackrel{0}{10}$ & $\dot{0}$ & मे & म & 7 & 20 & $\dddot{0}$ & $\begin{array}{l}0 \\
20\end{array}$ & 5 & $\overbrace{10}$ & मे. & ? \\
\hline - & $\underset{\infty}{\infty}$ & $\underbrace{0}_{20}$ & $\stackrel{\infty}{\leftarrow}$ & $\infty$ & $\stackrel{20}{4}$ & i⿱ & $\begin{array}{l}0 \\
10\end{array}$ & ف̊ & $\stackrel{20}{4}$ & $\begin{array}{l}\infty \\
1 \\
1\end{array}$ & 10. & $\dot{\infty}$ & $\frac{0}{0}$ \\
\hline$\forall$ है & $\begin{array}{l}8 \\
\vdots \\
0 \\
\dot{y} \\
1\end{array}$ & $\begin{array}{l}8 \\
\vdots \\
0\end{array}$ & $\begin{array}{l}8 \\
\dot{8} \\
\dot{8}\end{array}$ & $\begin{array}{l}8 \\
\vdots \\
\text { ๙ิ }\end{array}$ & $\begin{array}{l}8 \\
\text { \& } \\
\text { ล }\end{array}$ & $\begin{array}{l}8 \\
8 \\
\dot{q}\end{array}$ & $\begin{array}{l}8 \\
8 \\
0\end{array}$ & $\begin{array}{l}\stackrel{8}{0} \\
\dot{0} \\
\text { ๙ }\end{array}$ & $\begin{array}{l}8 \\
8 \\
8 \\
0\end{array}$ & $\begin{array}{l}8 \\
\vdots \\
\dot{\infty}\end{array}$ & $\begin{array}{l}8 \\
8 \\
\dot{0}\end{array}$ & $\begin{array}{l}8 \\
8 \\
0 \\
0 \\
\text { ปิ }\end{array}$ & 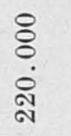 \\
\hline$\approx E$ & A & $\Rightarrow$ & $\Rightarrow$ & $\Rightarrow$ & $\Rightarrow$ & $\Rightarrow$ & $\Rightarrow$ & $\Rightarrow$ & $\Rightarrow$ & $\Rightarrow$ & $\Rightarrow$ & $\Rightarrow$ & $\Rightarrow$ \\
\hline 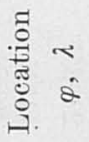 & 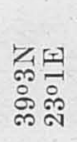 & 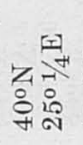 & 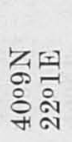 & 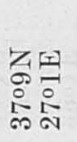 & 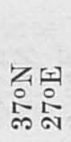 & 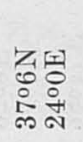 & 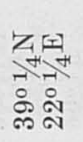 & 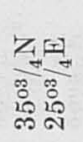 & 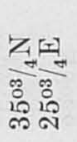 & 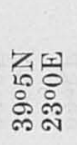 & 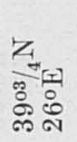 & 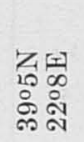 & 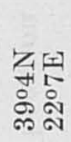 \\
\hline 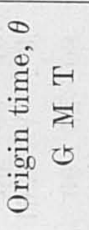 & 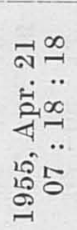 & 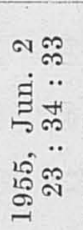 & 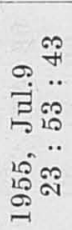 & 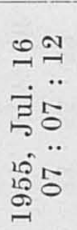 & 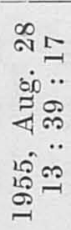 & 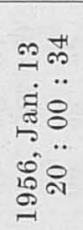 & 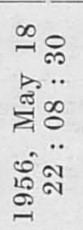 & 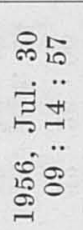 & 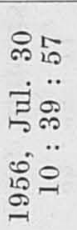 & 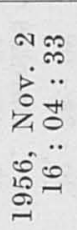 & 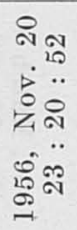 & 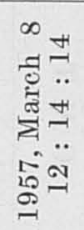 & 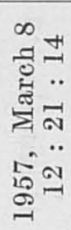 \\
\hline 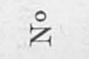 & $\&$ & $\bar{\sigma}$ & ๙ี & ๓ & 今 & 10 & $\mathscr{\sigma}$ & 5 & $\stackrel{\infty}{\circ}$ & $\stackrel{8}{\circ}$ & 8 & 후 & $\stackrel{\text { จิ }}{\varrho}$ \\
\hline
\end{tabular}




\begin{tabular}{|c|c|c|c|c|c|c|c|c|c|c|c|c|c|}
\hline 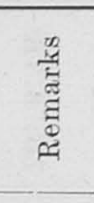 & 1 & $\begin{array}{l}\frac{1}{\infty} \\
10\end{array}$ & $\frac{1}{7}$ & $\begin{array}{l}\ddot{H} \\
\therefore \\
\ddot{0} \\
0 \\
0\end{array}$ & 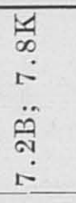 & $\frac{4}{10}$ & 1 & 1 & 1 & $\begin{array}{l}\qquad 1 \\
\infty \\
\dot{H}\end{array}$ & $\frac{11}{10}$ & $\frac{11}{20}$ & $\begin{array}{l}\frac{14}{21} \\
\frac{1}{10}\end{array}$ \\
\hline $\begin{array}{l}\stackrel{*}{\sharp} \\
\dot{\sharp}\end{array}$ & $\begin{array}{l}? \\
0 \\
1\end{array}$ & $\begin{array}{l}-1 \\
\dot{0} \\
+\end{array}$ & $\begin{array}{l}0 \\
0 \\
1\end{array}$ & $\begin{array}{l}\ddot{0} \\
+ \\
+\end{array}$ & $\begin{array}{l}\dddot{0} \\
+\end{array}$ & $\begin{array}{l}0 \\
0 \\
1\end{array}$ & $\begin{array}{l}+1 \\
0 \\
1\end{array}$ & $\begin{array}{l}1 \\
0 \\
1\end{array}$ & $\begin{array}{c}-1 \\
0 \\
1\end{array}$ & $\begin{array}{l}\stackrel{+}{0} \\
+\end{array}$ & $\begin{array}{l}4 \\
0 \\
0\end{array}$ & $\begin{array}{l}0 \\
0 \\
+\end{array}$ & $\begin{array}{c}-1 \\
1\end{array}$ \\
\hline$\stackrel{*}{\exists}$ & $\begin{array}{l}\infty \\
\infty \\
10\end{array}$ & \begin{tabular}{l}
$\infty$ \\
\multirow{1}{*}{} \\
$\stackrel{0}{0}$
\end{tabular} & $\begin{array}{l}\infty \\
\infty \\
i \infty\end{array}$ & 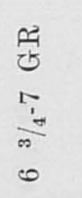 & $\begin{array}{l}\stackrel{2}{5} \\
\stackrel{2}{\circ} \\
\stackrel{2}{\circ}\end{array}$ & $\begin{array}{l}\infty \\
10 \\
20\end{array}$ & $\begin{array}{l}11 \\
\text { is }\end{array}$ & $\begin{array}{l}\infty \\
\sim \\
0\end{array}$ & $\begin{array}{l}\infty \\
\infty \\
10\end{array}$ & $\begin{array}{l}\infty \\
10 \\
10\end{array}$ & $\begin{array}{l}n \\
0 \\
10\end{array}$ & $\begin{array}{l}n \\
20 \\
20\end{array}$ & $\begin{array}{l}n \\
10 \\
10\end{array}$ \\
\hline ㄱ & 19. & ?. & $\stackrel{9}{4}$ & $\stackrel{?}{\because}$ & $\stackrel{4}{\check{1}}$ & में & $\stackrel{0}{10}$ & 10. & 10 & is. & is & $\stackrel{0}{10}$ & $\begin{array}{l}0 \\
10\end{array}$ \\
\hline - & فُ & $\stackrel{\infty}{\stackrel{\infty}{亡}}$ & $\begin{array}{l}0 \\
10\end{array}$ & $\stackrel{\infty}{i}$ & $\stackrel{\circ}{\circ}$ & $\stackrel{10}{4}$ & $\begin{array}{l}0 \\
\text { Lे }\end{array}$ & id & $\begin{array}{l}0 \\
\text { id }\end{array}$ & id & if & 5 & $\begin{array}{l}0 \\
\text { id }\end{array}$ \\
\hline 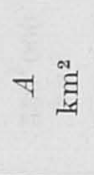 & $\begin{array}{l}0 \\
8 \\
0 \\
0\end{array}$ & $\begin{array}{l}8 \\
8 \\
\text { ลิ }\end{array}$ & $\begin{array}{l}8 \\
8 \\
\text { in } \\
\text { ज. }\end{array}$ & $\begin{array}{l}8 \\
8 \\
8 \\
\dot{8} \\
0 \\
-1 \\
-1\end{array}$ & $\begin{array}{l}8 \\
8 \\
0 \\
8 \\
\stackrel{-}{-1} \\
-1\end{array}$ & $\begin{array}{l}8 \\
\vdots \\
0 \\
1\end{array}$ & $\begin{array}{l}8 \\
\dot{8} \\
\dot{0}\end{array}$ & $\begin{array}{l}8 \\
8 \\
0 \\
0\end{array}$ & $\begin{array}{l}\stackrel{8}{0} \\
\dot{0} \\
=\end{array}$ & $\begin{array}{l}8 \\
8 \\
\dot{0} \\
\text { स1 }\end{array}$ & $\begin{array}{l}8 \\
8 \\
0 \\
10\end{array}$ & $\begin{array}{l}8 \\
8 \\
0 \\
0\end{array}$ & $\begin{array}{l}8 \\
8 \\
0 \\
\infty\end{array}$ \\
\hline$\approx I$ & $\Rightarrow$ & $\Rightarrow$ & $\nexists$ & $\widehat{\Xi}$ & $\widehat{\Xi}$ & $\Rightarrow$ & $\Rightarrow$ & $\Rightarrow$ & $\Rightarrow$ & $\Rightarrow$ & $\Rightarrow$ & $\Rightarrow$ & $\Rightarrow$ \\
\hline 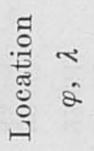 & 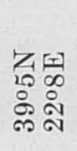 & 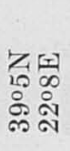 & 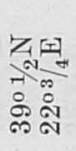 & 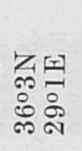 & 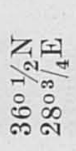 & 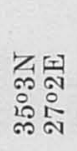 & 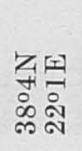 & 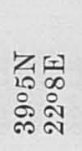 & 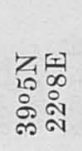 & 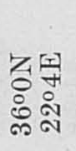 & 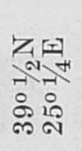 & 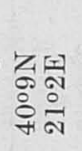 & 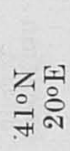 \\
\hline 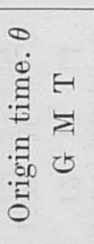 & 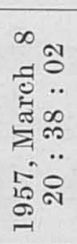 & 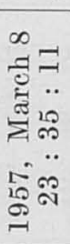 & 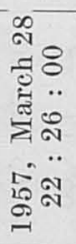 & 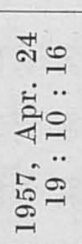 & 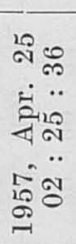 & 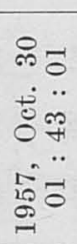 & 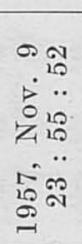 & 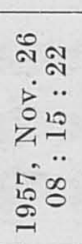 & 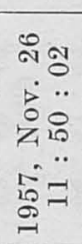 & 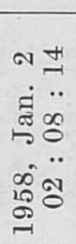 & 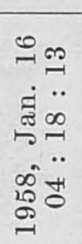 & 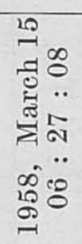 & 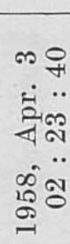 \\
\hline 文 & $\stackrel{\Re}{\stackrel{-}{*}}$ & $\underset{\sim}{\stackrel{+}{0}}$ & $\stackrel{20}{\stackrel{0}{-1}}$ & $\stackrel{\overbrace{}}{\circ}$ & $\stackrel{5}{\varrho}$ & $\stackrel{\infty}{\stackrel{\rho}{\varrho}}$ & $\stackrel{\circ}{\stackrel{0}{0}}$ & $\stackrel{=}{\exists}$ & $\exists$ & $\stackrel{\vartheta}{\exists}$ & $\stackrel{9}{=}$ & $\underset{\exists}{\exists}$ & $\stackrel{10}{=1}$ \\
\hline
\end{tabular}


Table 4 (cont.).

\begin{tabular}{|c|c|c|c|c|c|c|c|c|c|}
\hline No & $\begin{array}{l}\text { Origin } \operatorname{tim} \theta, \theta \\
\quad \text { G M T }\end{array}$ & $\begin{array}{c}\text { Location } \\
\qquad \varphi, \lambda\end{array}$ & $\begin{array}{c}h \\
\mathrm{~km}\end{array}$ & $\begin{array}{c}A \\
\mathrm{~km}^{2}\end{array}$ & $I_{0}$ & $M$ & $M^{*}$ & $M \cdot M^{*}$ & Remarks \\
\hline 116 & $\begin{array}{r}1958, \text { May } 9 \\
02: 40: 47\end{array}$ & $\begin{array}{l}36^{\circ} 1 / 2 \mathrm{~N} \\
27^{\circ} 3 / 4 \mathrm{E}\end{array}$ & n & 200.000 & $5-6$ & 6.1 & $5.4 \mathrm{~B}$ & +0.7 & - \\
\hline 117 & $\begin{array}{r}\text { 1958, Jul. } 17 \\
05: 37: 08\end{array}$ & $\begin{array}{l}40^{03} / 4 \mathrm{~N} \\
23^{01 / 4} \mathrm{E}\end{array}$ & $\mathbf{n}$ & 100.000 & 6.7 & 5.6 & $51 / 2 \mathrm{~K}$ & +0.1 & - \\
\hline 118 & $\begin{array}{l}\text { 1958, Aug. } 27 \\
15: 16: 34\end{array}$ & $\begin{array}{l}37^{\circ} 8 \mathrm{~N} \\
20^{\circ} 5 \mathrm{E}\end{array}$ & (n) & 900.000 & $5-6$ & 7.0 & $6.5 \mathrm{~B}$ & +0.5 & - \\
\hline 119 & $\begin{array}{c}1959, \text { Apr. } 25 \\
00: 26: 41\end{array}$ & $\begin{array}{l}37^{\circ} 0 \mathrm{~N} \\
28^{\circ} 5 \mathrm{E}\end{array}$ & $\mathbf{n}$ & 80.000 & $7-8$ & 5.7 & $6.3 \mathrm{~B}$ & -0.6 & - \\
\hline 120 & $\begin{array}{c}1959, \text { May } 14 \\
06: 36: 55\end{array}$ & $\begin{array}{l}35^{\circ 1 / 4} \mathrm{~N} \\
24^{03 / 4} \mathrm{E}\end{array}$ & n & 450.000 & $8-9$ & 6.8 & $61 / 2$ GR & +0.3 & $\begin{array}{c}6.2 \mathrm{~B} \\
6-61 / 4 \mathrm{~K}\end{array}$ \\
\hline 121 & $\begin{array}{c}\text { 1959, Jun. } 10 \\
04: 16: 03\end{array}$ & $\begin{array}{l}35^{\circ 3} / 4 \mathrm{~N} \\
24^{\circ} 1 / 4 \mathrm{E}\end{array}$ & n & 210.000 & $6-7$ & 6.2 & $5.7 \mathrm{~B}$ & +0.5 & - \\
\hline 122 & $\begin{array}{c}\text { 1959, Jul. } 26 \\
17: 07: 03\end{array}$ & $\begin{array}{l}41^{\circ \mathrm{N}} \\
27^{\circ 1 / 2 \mathrm{E}}\end{array}$ & $\mathrm{n}$ & 40.000 & $4-5$ & 5.0 & $5.1 \mathrm{~B}$ & -0.1 & - \\
\hline 123 & $\begin{array}{r}1959, \text { Aug. } 16 \\
18: 42: 00\end{array}$ & $\begin{array}{l}3701 / 4 \mathrm{~N} \\
22^{\circ} 0 \mathrm{~N}\end{array}$ & n & 90.000 & $6-7$ & 5.7 & $5.5 \mathrm{~B}$ & +0.2 & - \\
\hline 124 & $\begin{array}{c}1959, \text { Aug. } 17 \\
01: 33: 14\end{array}$ & $\begin{array}{l}41^{\circ} \mathrm{N} \\
19^{0} 1 / 2 \mathrm{E}\end{array}$ & $\mathbf{n}$ & 330.000 & $4-5$ & 6.3 & $5.8 \mathrm{~B}$ & +0.5 & $6 \mathrm{~K}$ \\
\hline
\end{tabular}


Table 5 - Elements of California Shocks from data of U S C G S

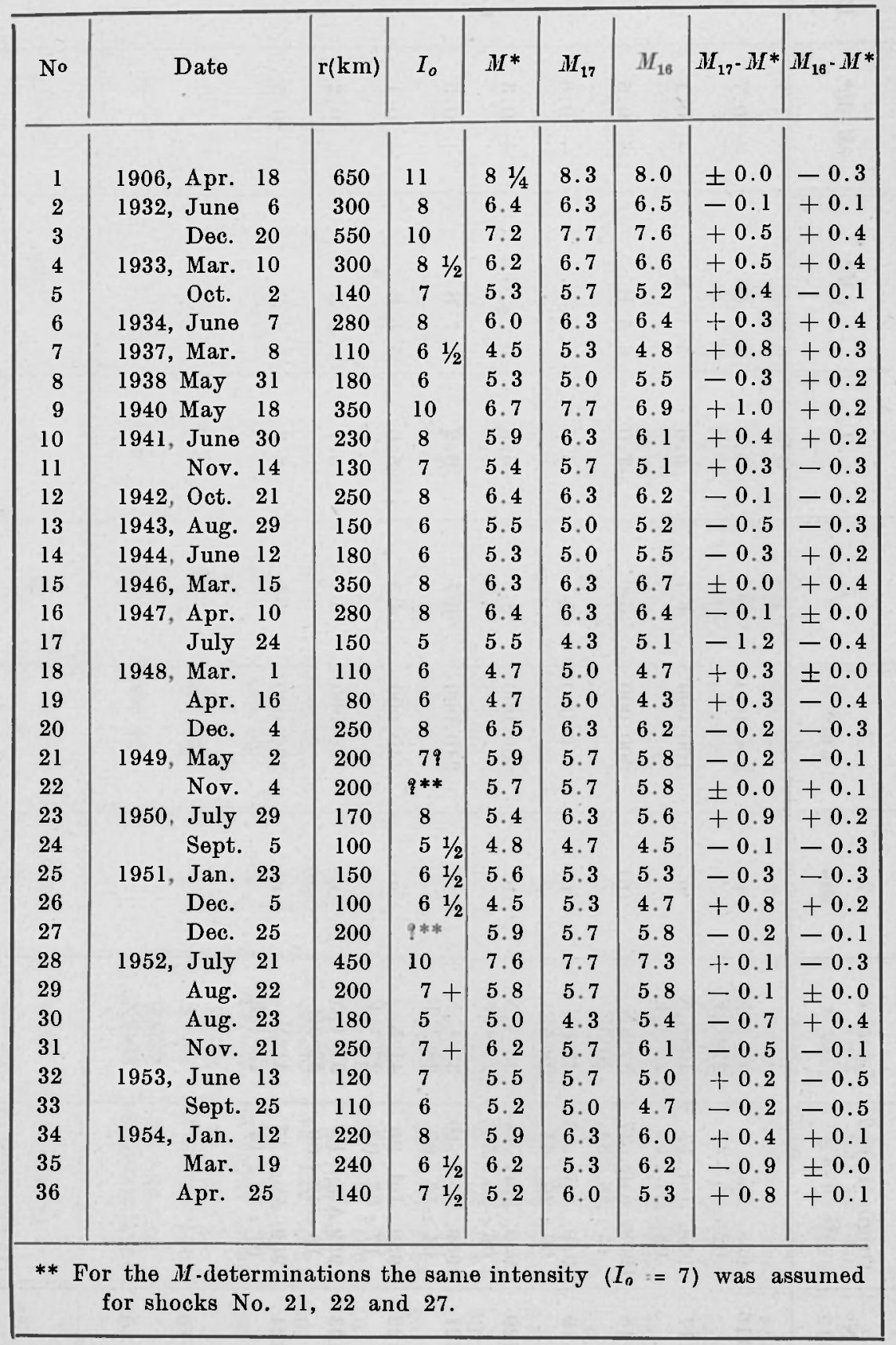


T able 6 - Magnitudes of California Shocks From data of U S C G S*

\begin{tabular}{|c|c|c|c|c|c|c|c|c|c|}
\hline No & $M^{*}$ & $M_{19}$ & $M_{21}$ & $M_{23}$ & $M_{24}$ & $M_{19} \cdot M^{*}$ & $M_{21}-M^{*}$ & $M_{23}-M^{*}$ & $M_{24}-M^{*}$ \\
\hline 1 & $8 \mathrm{I} / 4$ & 7.7 & 7.6 & 9.4 & 9.5 & -0.6 & -0.7 & $+1 \cdot 1$ & +1.2 \\
\hline 2 & 6.4 & 6.2 & 6.5 & 7.6 & 7.6 & -0.2 & +0.1 & +1.2 & +1.2 \\
\hline 3 & 7.2 & 7.3 & 7.4 & 8.9 & 8.9 & +0.1 & +0.2 & +1.7 & +1.7 \\
\hline 4 & 6.2 & 6.3 & 6.5 & 7.8 & 7.8 & +0.1 & +0.3 & +1.6 & +1.6 \\
\hline 5 & 5.3 & 5.3 & 5.5 & 6.4 & 6.4 & \pm 0.0 & +0.2 & +1.1 & +1.1 \\
\hline 6 & 6.0 & 6.1 & 6.4 & 7.5 & 7.5 & +0.1 & +0.4 & +1.5 & +1.5 \\
\hline 7 & 4.5 & 4.9 & 5.2 & 6.1 & 6.0 & +0.4 & +0.7 & +1.6 & +1.5 \\
\hline 8 & 5.3 & 5.2 & 5.7 & 6.3 & 6.3 & -0.1 & +0.4 & +1.0 & +1.0 \\
\hline 9 & 6.7 & 6.9 & 6.8 & 8.4 & 8.5 & +0.2 & +0.1 & +1.7 & +1.8 \\
\hline 10 & 5.9 & 6.0 & 6.2 & 7.3 & 7.3 & +0.1 & $+c$ & +1.4 & +1.4 \\
\hline 11 & 5.4 & 5.2 & 5.4 & 6.4 & 6.4 & -0.2 & \pm 0.0 & +1.0 & +1.0 \\
\hline 12 & 6.4 & 6.0 & 6.3 & 7.4 & 7.4 & -0.4 & -0.1 & +1.0 & +1.0 \\
\hline 13 & 5.5 & 5.0 & 5.5 & 6.2 & 6.1 & -0.5 & \pm 0.0 & +0.7 & +0.6 \\
\hline 14 & 5.3 & 5.2 & 5.7 & 6.3 & 6.3 & -0.1 & +0.4 & +1.0 & +1.0 \\
\hline 15 & 6.3 & 6.3 & 6.7 & 7.7 & 7.7 & \pm 0.0 & +0.4 & +1.4 & +1.4 \\
\hline 16 & 6.4 & 6.1 & 6.4 & 7.5 & 7.5 & -0.3 & \pm 0.0 & +1.1 & +1.1 \\
\hline 17 & 5.5 & 4.8 & 5.4 & 5.8 & 5.8 & -0.7 & -0.1 & +0.3 & +0.3 \\
\hline 18 & 4.7 & 4.8 & 5.1 & 5.9 & 5.8 & +0.1 & +0.4 & +1.2 & +1.1 \\
\hline 19 & 4.7 & 4.5 & 4.7 & 5.6 & 5.5 & -0.2 & .0 & +0.9 & +0.8 \\
\hline 20 & 6.5 & 6.0 & 6.3 & 7.4 & 7.4 & -0 & -0.2 & +0.9 & +0.9 \\
\hline 21 & 5.9 & 5.6 & 5.9 & 6.8 & 6.8 & -0.3 & \pm 0.0 & +0.9 & +0.9 \\
\hline 22 & 5.7 & 5.6 & 5.9 & 6.8 & 6.8 & -0.1 & +0.2 & +1.1 & +1.1 \\
\hline 23 & 5.4 & 5.7 & 5.8 & 7.0 & 7.0 & +0.3 & +0.4 & +1.6 & +1.6 \\
\hline 24 & 4.8 & 4.6 & 4.9 & 5.6 & 5.5 & -0.2 & +0.1 & +0.8 & +0.7 \\
\hline 25 & 5.6 & 5.2 & 5.5 & 6.4 & 6.3 & -0.4 & -0.1 & +0.8 & +0.7 \\
\hline 26 & 4.5 & 4.9 & 5.0 & 6.0 & 5.9 & +0.4 & +0.5 & +1.5 & +1.4 \\
\hline 27 & 5.9 & 5.6 & 5.9 & 6.8 & 6.8 & -0.3 & \pm 0.0 & +0.9 & +0.9 \\
\hline 28 & 7.6 & 7.1 & 7.1 & 8.7 & 8.7 & -0.5 & -0.5 & +1.1 & +1.1 \\
\hline 29 & 5.8 & 5.6 & 5.9 & 6.8 & 6.8 & -0.2 & +0.1 & +1.0 & +1.0 \\
\hline 30 & 5.0 & 4.9 & 5.6 & 6.0 & 5.9 & -0.1 & +0.6 & +1.0 & +0.9 \\
\hline 31 & 6.2 & 5.7 & 6.2 & 7.0 & 7.0 & -0.5 & \pm 0.0 & +0.8 & +0.8 \\
\hline 32 & 5.5 & 5.2 & 5.3 & 6.3 & 6.3 & -0.3 & -0.2 & +0.8 & +0.8 \\
\hline 33 & 5.2 & 4.8 & 5.1 & 5.9 & 5.8 & -0.4 & -0.1 & +0.7 & +0.6 \\
\hline 34 & 5.9 & 5.9 & 6.1 & 7.3 & 7.3 & \pm 0.0 & +0.2 & +1.4 & +1.4 \\
\hline 35 & 6.2 & ธ. 6 & 6.1 & 6.8 & 6.8 & -0.6 & -0.1 & +0.6 & +0.6 \\
\hline 36 & 5.2 & 5.4 & 5.5 & 6.7 & 6.6 & +0.2 & +0.3 & +1.5 & +1.4 \\
\hline
\end{tabular}

* For the $M$-determinations the epicentral intensity $I_{0}$ and the radius of perceptibility $r$ were taken from Table 5 . 


\section{$S U M M A R Y$}

A simple magnitude formula for macroseismic data is proposed which places the $M$-determination on a self-consistent and independent basis, with the great advantage of being equally reliable to the $M$-determination from instrumental data and directly applicable to shocks of any focal depth, and very probably of any country.

\section{ZUSAM MENFASSUNG}

Es wird eine einfache Magnitudengleichung fur makroseismische Daten vorschlägt, wodurch die Magnitudenbestimmung auf einer selbskonsequenten und selbstständigen Grundlage gesetzt wird, mit dem grossen Vorteil diese Bestimmung mit derjenigen aus milkroseismischen Daten gleichermassen zuverlässig und geradeswegs auf Beben irgendeiner Herdtiefe und sehr wahrscheinlich irgendeines Gebietes anwendbar zu sein.

\section{$R E S U M E$}

On propose une formule simple pour la determination de la magnitude des séismes par les données macroséismiques, qui est directement applicable aux séismes de toutes profondeurs et très probablement de toutes régions. $L$ a determination macroséismique de $M$ par cette méthode ale grand avantage d'être aussi valable que la determination de.M par les données des instruments.

\section{RIASSUNTO}

Viene proposta una formula semplice per la determinazione della magnitudine $M$ dai dati macrosismici, la quale formula è direttamente applicabile ai terremoti di ogni profondità e molto probabilmente di ogni regione. $L$ a determinazione macrosismica dell' $M$ con il metodo suddetto ha il grande vantaggio di essere talmente valevole quanto la determinazione dell' $M$ dai dati degli strumenti. 


\section{REFERENCES}

ВАтті M., Seismicity of Fennoscandia and Related Problems, in "Gerl. Beitr. z. Geoph. ", 63, 3, 173-208, (1953).

- The Problem of Earthquake Magnitude Determination, in "Publ. du B. C. S. I., Serie A, Trav. Sc. ", Fasc. 19, 5-63, (1956a).

- Some Consequences of the Existence of Low-Telocity Layers, in "Ann. di Geof. ", 9, 4, 411-450, (1956b).

- The Energies of Seismic Body Waves and Surface Waves, iu "Contr. in. Geoph. in Honor of Beno Gutenberg ", Pergamon Press, 1-16, (1958).

Benioff H.-Gutenberg B., General Introduction to Seismology, in "Earthquakes in Kern Country, California during 1952 ", State of Calif., Division of Mines, "Bull. ", 171, 131-135, (1955).

Byerly P., Seismology, New York, 71, (1942).

BXERLY P. - DeNOYER J., Energy in Earthquales as Computed from Geodetic Observations, in "Contr. in Geoph. in Honor of Beno Gutenberg ", Pergamon Press, 17-35, (1958).

Eiby G., About Earthquakes, New York, 24, (1957).

Galanopoulos A., Greece, A Catalogue of Shocks with Io $>$ VI or $M \gg 5$ for the Years 1801-1958, Athens, (1960).

Gutenberg B., Magnitude Determination for Deep-Focus Earthquakes, in "Bull. Seism. Soc. Am. ", 35, 3, 117-130, (1945).

- Great Earthquakes 1896-1903, in "Trans. Am. Geoph. Union" 37, 5, 608-614, (1956).

- Major Earthquakes of 1957, in "Bull. Seism. Soc. Am. ", 49, 44, 422, (1959).

Gutenberg B. - Richter C., Earthquake Magnitude, Intensity, Energy, and Acceleration, in "Bull. Seism. Soc. Am. ", 32, 3, 163-191, (1942).

- Earthyuake Magnitude, Intensity, Energy, and Acceleration, in "Bull. Seism. Soc. Am. 》, 46 2, 105-145, (1956a).

- Magnitude and Energy of Earthquakes, in "Ann. di Geof.", 9, 1, $1-15,(1956 \mathrm{~b})$.

Hodgson J.-Stevens A., Direction of Faulting in Some of the Larger Earthquakes of 1955-1956, in "Publ. Dom. Obs. ", 19, 281-317, (1958).

KaRNIK V., Magnitudenbestimmung Europäischer Nahbeben, in "Trav. Inst. Geoph. Acad. Tehec. Scien. ", 47, 399-522, (1956).

- Seismicity of Europe. A Progress Report Presented at the Helsinki Meeting of the E. S. C. July 25, 1960, Phaha, (1960).

Richter C., Elementary Seismology, San Francisco, (1958).

Sieberg A., Erdebengeologie, in "Gutenbergs Handbuch der Geophysik ", 4, Lif. 2, Berlin, (1932).

SponhedeR W., Methoden zur Herdtiefenbestimmung in der Makroseismik, in "Freib. Forsch.-H.», C 88, 1-120, (1960).

Toperczer M., Zur Definition der Seismizät, in "Arch. Met. Geoph. Biokl. », A, 5, 4, 377-385, (1953).

- Lehrbuch der Allgemeinen Geophysik, Wien, 250, (1960). 